COMMUNICATIONS IN

ANALYSIS AND GEOMETRY

Volume 8, Number 5, 969-1026, 2000

\title{
Higher eigenvalues and isoperimetric inequalities on Riemannian manifolds and graphs
}

\author{
Fan Chung ${ }^{1}$, AleXander Grigor'yan ${ }^{2}$ and Shing-Tung YaU ${ }^{3}$
}

\section{Contents.}

1 Introduction . . . . . . . . . . . . . . . . . . . . . 969

2 Isoperimetric inequality for weighted manifolds . . . . . . 9976

3 Eigenvalues of subsets . . . . . . . . . . . . . . . . .984

4 Sobolev inequality . . . . . . . . . . . . . . .993

5 Analysis on weighted graphs . . . . . . . . . . . .997

5.1 Measures on graphs . . . . . . . . . . . . . . 997

5.2 Discrete Laplacian . . . . . . . . . . . . . . . . 998

5.3 Green's formula . . . . . . . . . . . . . . . . . . . 999

5.4 Integration versus Summation . . . . . . . . . . . 999

5.5 Eigenvalues of Laplacian . . . . . . . . . . . . . . . . . 1000

5.6 Heat kernel . . . . . . . . . . . . . . . . . . . . . . 1000

5.7 Co-area formula . . . . . . . . . . . . . . . . . . . 1001

6 Discrete isoperimetric inequality . . . . . . . . . . . . . 1002

6.1 Statement of the results . . . . . . . . . . . . . . . . 1002

6.2 Boundary of a ball . . . . . . . . . . . . . . . . . . 1010

6.3 Proof of isoperimetric inequality . . . . . . . . . . . 1013

7 Eigenvalues and Sobolev inequality on graphs . . . . . . . . 1019

\section{Introduction.}

The purpose of this paper is to demonstrate in a rather general setup how isoperimetric inequalities and lower bounds of the eigenvalues of the Laplacian can be derived from existence of a distance function with controllable

\footnotetext{
${ }^{1}$ Research supported in part by NSF Grant No. DMS 98-01446

${ }^{2}$ Supported by EPSRC Fellowship B/94/AF/1782

${ }^{3}$ Supported in part by NSF Grant No. DMS 95-04834
} 
Laplacian. For $x \in \mathbb{R}^{n}$, let us denote $\rho(x)=|x|=\left(\sum_{i} x_{i}^{2}\right)^{1 / 2}$. It is obvious that we have the following two relations

$$
\begin{aligned}
\Delta\left(\rho^{2}\right) & =2 n, \\
|\nabla \rho| & =1, \quad x \neq 0 .
\end{aligned}
$$

By integrating (1.1) over the ball $B(r)$ of radius $r$ centered at the origin, we obtain

$$
2 n \operatorname{Vol}(B(r))=\int_{B(r)} \Delta\left(\rho^{2}\right) d \operatorname{Vol}(x)=\int_{\partial B(r)} 2 \rho \frac{\partial \rho}{\partial \nu} d \mathrm{~A}=2 r \mathrm{~A}(\partial B(r))
$$

where we have used the fact that on the boundary $\frac{\partial \rho}{\partial \nu}=|\nabla \rho|=1$. Therefore, we have the following identity for the volume function $V(r):=\operatorname{Vol}(B(r))$

$$
V(r)=\frac{n}{r} V^{\prime}(r) .
$$

Of course, the relation (1.3) of the volume and the boundary area of the Euclidean ball is well known from the elementary geometry. However, (1.1)(1.2) can also be used in a rather sophisticated way to prove the following isoperimetric inequality between the volume and the boundary area of any bounded region $\Omega \subset \mathbb{R}^{n}$ (assume for simplicity that the boundary $\partial \Omega$ is smooth)

$$
\mathrm{A}(\partial \Omega) \geq c \operatorname{Vol}^{1-1 / n}(\Omega) .
$$

The constant $c$ obtained in this way, is not the sharp one. As is well-known, the exact constant $c$ in (1.4) is one for which both sides of (1.4) coincide for $\Omega$ being a ball.

The present work makes consistent use of the hypothesis that, on a Riemannian manifold $M$, there exists a distance function ${ }^{4} \rho_{\xi}(x)$ possessing an upper bound for $\left|\nabla \rho_{\xi}\right|$ and a lower bound for $\Delta \rho_{\xi}^{2}$, where $\nabla$ and $\Delta$ are the Riemannian gradient and Laplacian, respectively. Given that much, we produce an isoperimetric inequality of type (1.4).

Furthermore, we derive from the isoperimetric inequality a lower bound for $\lambda_{k}(\Omega)$ being the $k$-th Dirichlet eigenvalue of the Laplace operator in a region $\Omega \subset M$. Here is one of our main results, which will be proved at the end of Section 3. Denote by $B_{\xi}(r)$ the open $\rho$-ball centered at $\xi \in M$ of radius $r$.

\footnotetext{
${ }^{4}$ Despite the distance is symmetric in $x, \xi$, we use the asymmetric notation to emphasize that $\rho_{\xi}(x)$ is always considered as a function of $x$, with a fixed $\xi$. Note that $\rho_{\xi}(x)$ is not necessarily the Riemannian distance.
} 
Theorem 1.1. Let $M$ be a complete Riemannian manifold of dimension $n>1$. Let $\rho_{\xi}(x)$ be a distance function on $M$ such that, for some $R_{0} \in$ $(0,+\infty]$ and all $\xi \in M$, the inequalities

$$
\left|\nabla \rho_{\xi}\right| \leq 1
$$

and

$$
\Delta \rho_{\xi}^{2} \geq 2 n
$$

hold inside the ball $B_{\xi}\left(R_{0}\right)$. Then, for any precompact open set $\Omega \subset M$ with smooth boundary and such that

$$
\operatorname{Vol}(\Omega) \leq \varepsilon R_{0}^{n},
$$

we have

$$
\mathrm{A}(\partial \Omega) \geq c \operatorname{Vol}(\Omega)^{1-1 / n},
$$

where $\varepsilon$ and $c$ are positive constants depending only on $n$.

Furthermore, if, for a positive integer $k$,

$$
\operatorname{Vol}(\Omega) \leq \varepsilon R_{0}^{n} k
$$

then

$$
\lambda_{k}(\Omega) \geq a\left(\frac{k}{\operatorname{Vol}(\Omega)}\right)^{2 / n}
$$

with some $a=a(n)>0$.

If $R_{0}=\infty$, that is, (1.5) and (1.6) hold all over $M$, then the restrictions (1.7) and (1.9) are void, and we get (1.8) for all $\Omega$ and (1.10) for all $\Omega$ and $k \geq 1$.

If $R_{0}<\infty$ then the isoperimetric inequality (1.8) is claimed to be true only for $\Omega$ with a small enough volume. However, whatever is $\operatorname{Vol}(\Omega)$, the condition (1.9) is satisfied for $k$ large enough, namely for

$$
k \geq \frac{\operatorname{Vol}(\Omega)}{\varepsilon R_{0}^{n}} .
$$

Therefore, the lower bound (1.10) is true for all $\lambda_{k}(\Omega)$ with such $k$.

The following smoothness of $\rho$ is assumed: $\rho_{\xi}$ is Lipschitz in $B_{\xi}\left(R_{0}\right)$ and $\rho_{\xi}^{2}$ is $C^{\infty}$ in $B_{\xi}\left(R_{0}\right)$. We can prove the same result assuming only that $\rho_{\xi}$ 
is Lipschitz in $B_{\xi}\left(R_{0}\right)$, provided (1.6) holds in the sense of distributions. However, in the latter case, the proof involves some technical complications which make the argument less transparent and which are avoided by taking $\rho_{\xi}$ being smooth enough.

Let us consider some examples when the hypotheses (1.5) and (1.6) hold.

Example 1.2. Let $\rho$ be the geodesic distance on $M$. Then (1.5) is satisfied automatically. Suppose that $M$ is a Cartan-Hadamard manifold, that is, a simply connected geodesically complete non-compact manifold with non-positive sectional curvature. The inequality (1.6) is satisfied, by the comparison theorem for the Laplacian (see [31]). Thus, Theorem 1.1 applies on such a manifold, with $R_{0}=\infty$.

The isoperimetric inequality (1.8) on Cartan-Hadamard manifolds was first proved by Hoffman and Spruck [19].

Example 1.3. Let $M$ be a $n$-dimensional minimal submanifold of $\mathbb{R}^{N}$ and let $\rho_{\xi}(x)$ be the Euclidean distance function in $\mathbb{R}^{N}$ restricted to $M$. The inequality (1.5) is trivially satisfied. The well-known fact that the coordinate functions in $\mathbb{R}^{N}$ are harmonic on $M$ (see, for example, [27]) implies that, for the Laplacian on $M$,

$$
\Delta \rho_{\xi}^{2}=2 n .
$$

Thus, all the hypotheses of Theorem 1.1 are satisfied.

The isoperimetric inequality on minimal surfaces was originally proved by Bombieri, De Giorgi and Miranda [2] (see also [1]). Michael and Simon [25] proved a similar result for more general submanifolds of $\mathbb{R}^{N}$.

Example 1.4. Let $M$ be a manifold with non-positive sectional curvature and let $R_{0}$ be its injectivity radius. Then (1.6) holds for the geodesic distance $\rho$. If, in addition, manifold $M$ is a compact then we may take $\Omega=M$ (the Dirichlet boundary condition disappears though when considering $\lambda_{k}(M)$ ) and obtain the lower bound (1.10) for $\lambda_{k}(M)$ provided

$$
k \geq \frac{\operatorname{Vol}(M)}{\varepsilon R_{0}^{n}} .
$$

Example 1.5. Let us show that one cannot in general obtain the estimate (1.10) for the lower eigenvalues. Indeed, let $M$ consist of $l$ disjoint copies of a compact $n$-dimensional manifold $K$ for which the hypotheses of Theorem 1.1 hold. For example, $K$ may be a flat torus, and $R_{0}$ may be its injectivity 
radius (see the previous example). Then (1.5) and (1.6) hold also on $M$ with the same $R_{0}$ and $\rho_{\xi}(x)$ (define $\rho_{\xi}(x)$ to be a huge constant when $x$ and $\xi$ belong to different copies of $K$ ). By Theorem 1.1, we have the lower bound (1.10) for $\lambda_{k}(M)$ provided $k$ satisfies (1.12).

On the other hand, for any $k \leq l$, we have $\lambda_{k}(M)=0$, because the eigenvalue 0 has the multiplicity $l$. Hence, the lower bound (1.10) fails if

$$
k \leq l=\frac{\operatorname{Vol}(M)}{\operatorname{Vol}(K)} .
$$

We see that the restriction (1.12) on $k$ has a correct (linear) order in $\operatorname{Vol}(M)$. Of course, one may obtain from this example a connected manifold $M$, by gluing all the copies of $K$ by thin tubes.

Denote by $p(t, x, y)$ the heat kernel of the Laplace operator on $M$ and by $h(B)$ the Cheeger constant of an open set $B \subset M$, that is,

$$
h(B):=\inf _{\Omega \subset \subset B} \frac{\mathrm{A}(\partial \Omega)}{\operatorname{Vol}(\Omega)}=\inf _{\substack{f \in C_{0}^{\infty}(B) \\ f \neq 0}} \frac{\int_{B}|\nabla f| d \mu}{\int_{B}|f| d \mu} .
$$

The proof of Theorem 1.1 is a combination of various arguments as shown on the following diagram (we omit all quantors for simplicity):

$$
\begin{aligned}
\Delta \rho_{\xi}^{2} & \geq 2 n \\
\Downarrow & \\
h\left(B_{\xi}(r)\right) & \geq \frac{n}{r} \\
\Downarrow & \\
\mathrm{A}(\partial \Omega) & \geq c \operatorname{Vol}(\Omega)^{1-1 / n} \\
& \Downarrow \\
\lambda_{1}(\Omega) & \geq \frac{c^{2}}{4} \operatorname{Vol}(\Omega)^{-2 / n} \\
& \Downarrow \\
p(t, x, y) & \leq C t^{-n / 2} \\
& \Downarrow \\
\lambda_{k}(\Omega) & \geq a k^{2 / n} \operatorname{Vol}(\Omega)^{-2 / n} .
\end{aligned}
$$

Our contribution here is twofold: 
1. the usage of the above chain of implications, which is new by itself (although most steps considered separately may be not new);

2. the proof of the implication (1.14) $\Longrightarrow(1.15)$ under the restriction (1.7).

For the latter, we use a localized (and simplified) version of the argument of Michael and Simon [25]. The hypothesis (1.14) contains already some initial lower bound for $\mathrm{A}(\partial \Omega)$ provided $\Omega$ lies in a ball of radius $R_{0}$. However, even if $\operatorname{Vol}(\Omega)$ is small as in (1.7), $\Omega$ may be long and thin and may be not contained in any such ball. To prove the isoperimetric inequality (1.15) for such $\Omega$, we use a certain ball covering argument - see Steps 2 and 3 of the proof of Theorem 2.1 below.

All other implications are relatively well-known, but we provide full proofs for the sake of completeness, especially as our setting does not altogether match those in the previous works. The part $(1.13) \Longrightarrow(1.14)$ is obtained simply by integration of (1.13) over $\Omega \subset B$ (see (2.10)). Inequality (1.14) can be restated as follows: for any $\Omega \subset B(r)$

$$
\mathrm{A}(\partial \Omega) \geq \frac{n}{r} \operatorname{Vol}(\Omega)
$$

which is a weak form of (1.15).

The part (1.15) $\Longrightarrow(1.16)$ is the well-known Cheeger argument [6] (see also [24])). It is implemented here as Step 1 of the proof of Theorem 3.1.

The part (1.16) $\Longrightarrow(1.17)$ is due to the second author [15] and Carron [3]. It is presented in Steps 2-3 of the proof of Theorem 3.1.

Finally, the part (1.17) $\Longrightarrow(1.18)$ uses the argument of Cheng and Li [8] (see also [15, Corollaries 2.1,2.2]) and constitutes Step 4 of the proof of Theorem 3.1. See also Corollary 3.3 for the final statement.

Let us mention that the sharp constant $a$ in $(1.10) /(1.18)$ is still unknown even for $\mathbb{R}^{n}$. According to the Pólya conjecture [28], it should be equal to the constant in the Weyl asymptotic formula. The best known $a$ in $\mathbb{R}^{n}$ is due to P.Li and the third author [20] (see also [21]).

In Section 4, we obtain, under the hypotheses of Theorem 1.1, a certain Sobolev inequality. We use a localized version of the argument of Federer and Fleming [14], [13] and Maz'ya [22], [23].

In the second part of the paper, we consider the discrete case. Previously, two of the authors [9] proved that under the assumption of

$$
\mathrm{A}(\partial \Omega) \geq c \operatorname{Vol}(\Omega)^{1-1 / n},
$$

for any finite subset $\Omega$ of a graph $\Gamma$, we can derive Sobolev inequalities for graphs and establish lower bounds for eigenvalues $\lambda_{k}$. 
However, obtaining the isoperimetric inequalities is not straightforward if we wish to start with a distance function in the spirit of Theorem 1.1. For example, the graph distance function, say, for the rectangular lattices $\mathbb{Z}^{n}$, does not satisfy (1.13). To circumvent this difficulty, we will consider more than one distance-like functions and we will introduce a notion of spring ratio $\nu$ (see definition (6.3) in Section 6). Here we state one of results that will be proved in Section 6 .

The underlying space in the discrete setting is a weighted graph $(\Gamma, \sigma)$ where $\sigma$ is a measure on the edge set $E$ of $\Gamma$. It induces also a measure $\mu$ on the vertex set $V$ of $\Gamma$ (see Section 5 for detailed definitions). Assume that there are a distance function $\rho_{\xi}(x)$ and a function $q_{\xi}(x)$ of pairs of vertices $x, \xi$ which satisfy the following hypotheses, with some positive constants $R_{0}, \delta$ and $\iota$ :

for any vertex $\xi$ and for all adjacent vertices $x, y \in B_{\xi}\left(R_{0}\right)$,

$$
\begin{aligned}
\left|\rho_{\xi}(x)-\rho_{\xi}(y)\right| & \leq 1, \\
\left|q_{\xi}(x)-q_{\xi}(y)\right| & \leq \rho_{\xi}(x)+\iota,
\end{aligned}
$$

and, for any $x \in B_{\xi}\left(R_{0}\right)$,

$$
\Delta q_{\xi}(x) \geq \delta
$$

Here $B_{\xi}(r)$ is the open $\rho$-ball centered at $\xi$ of radius $r$, and $\Delta$ is the discrete Laplace operator associated with the weight $\sigma$.

Furthermore, assume that

$$
n:=\delta \nu_{R_{0}+1} \geq 1,
$$

where the spring ratio $\nu_{r}$ is as defined in (6.3). Then, for any subset $\Omega \subset V$ such that $\mu(\Omega) \leq \varepsilon R_{0}^{n}$, we have

$$
\sigma(\partial \Omega) \geq c \mu(\Omega)^{1-1 / n}
$$

where $c$ and $\varepsilon$ are positive constants depending on the hypotheses (see Theorem 6.3).

The analogue of the function $q$ in the case of manifolds would be $\frac{1}{2} \rho^{2}$, in which case (1.13) would become (1.20) with $\delta=n$. However, even for the rectangular lattice $\mathbb{Z}^{n}$, for all reasonable choices of $\rho$ and $q$, the number $\delta$ in (1.20) is smaller than $n$. An additional argument with the spring ratio is required to recover the isoperimetric dimension $n$. 
Under the above hypotheses, we obtain also the eigenvalues estimates analogous to Theorem 1.1. Most proofs in the graph case are similar to those in the case of manifolds. In Sections 6 and 7, we pay special attention to the differences between the discrete and continuous settings.

Acknowledgments. This work was done during the second author's visit of the Harvard University. He is grateful to the Mathematics Department of this university for its hospitality. He also acknowledges support of the EPSRC (England).

\section{Isoperimetric inequality for weighted manifolds.}

By a weighted manifold, we call a pair $(M, \mu)$ of a Riemannian manifold $M$ and a Radon measure $\mu$ on $M$. We always assume that measure $\mu$ has a smooth strictly positive density $\eta(x)$ with respect to the Riemannian measure. It is well known that a Riemannian structure induces an associated Laplace operator. Similarly, a weighted manifold possesses a natural weighted Laplace operator defined by

$$
\Delta f:=\eta^{-1} \operatorname{div}(\eta \nabla f)
$$

where $\nabla$ and div are the Riemannian gradient and divergence, respectively. It is easy to see that $\Delta$ is formally self-adjoint with respect to measure $\mu$. If $\eta \equiv 1$ then $\Delta$ is the Riemannian Laplace operator.

Apart from having measure $\mu$ on $M$, we assume that $M$ is endowed with a distance function $\rho_{\xi}(x)$ between the points $x, \xi \in M$ (which is not necessarily the Riemannian distance). This function should satisfy all usual axioms of the distance. We use the asymmetric notation for the distance to facilitate considering it as a function of $x$, with a fixed $\xi$. Denote by

$$
B_{\xi}(r):=\left\{x \in M: \rho_{\xi}(x)<r\right\}
$$

balls associated with $\rho_{\xi}(x)$.

We always assume that $\rho_{\xi}(x)$ is Lipschitz as a function of $x$ and that $\rho_{\xi}^{2}$ is $C^{\infty}$-smooth in $x$, provided $x$ varies in $B_{\xi}\left(R_{0}\right)$ with a fixed radius $R_{0}$. For example, if $\rho_{\xi}(x)$ is the Riemannian distance then these assumptions hold provided $R_{0}$ is smaller than the injectivity radius of $M$ (which ensures that $x$ is away from the cut locus of $\xi$ ).

\section{Other notation:}


$\sigma$ - the boundary area which has the density $\eta$ with respect to the Riemannian Hausdorff measure of codimension 1;

$\lambda_{k}(\Omega)$ - the $k$-th Dirichlet eigenvalue of $-\Delta$ in the region $\Omega \subset M$.

Theorem 2.1. Let $M$ be a geodesically complete manifold. Assume that there exists $R_{0}>0$ such that, for any $\xi \in M$, the following inequalities hold in the ball $B_{\xi}\left(R_{0}\right)$ :

$$
\left|\nabla \rho_{\xi}\right| \leq 1
$$

and

$$
\Delta \rho_{\xi}^{2} \geq 2 n,
$$

with some constant $n>1$. Assume also that, for any $\xi \in M$, we have

$$
\lim \sup _{R \rightarrow 0} \frac{\mu\left(B_{\xi}(R)\right)}{R^{n}} \geq \omega
$$

with some constant $\omega>0$. Let $\Omega$ be an open subset of $M$ with smooth boundary such that

$$
\mu(\Omega) \leq \frac{\omega}{5} R_{0}^{n}
$$

Then

$$
\sigma(\partial \Omega) \geq c \mu(\Omega)^{1-1 / n}
$$

where

$$
c=2^{1-2 n}\left(\frac{\omega}{5}\right)^{1 / n}
$$

Remark 2.2. Constant $c$ is not claimed to be sharp.

Remark 2.3. Manifold $M$ may be not geodesically complete. However, $\Omega$ must be then precompact. More precisely, we need in the proof that the intersection of $\Omega$ with any ball is precompact.

Remark 2.4. Condition (2.3) holds automatically if $n$ is the dimension of $M$ and if $\mu$ is the Riemannian measure. However, in general, condition (2.3) cannot be dropped. Indeed, let $M=\mathbb{R}^{l}$ with $l>n$ and let $\rho_{\xi}(x)=|x-\xi|$. Then $\Delta \rho_{\xi}^{2}=2 l>2 n$ so that $(2.2)$ is satisfied. However, $\frac{\mu\left(B_{\xi}(R)\right)}{R^{n}} \rightarrow 0$ as $R \rightarrow 0$, and (2.3) fails. Clearly, the isoperimetric inequality (2.5) does not hold, for example, when $\Omega$ is a ball, in which case we have

$$
\sigma(\partial \Omega) \sim \mu(\Omega)^{1-1 / l}=o\left(\mu(\Omega)^{1-1 / n}\right) \quad \text { as } \quad \mu(\Omega) \rightarrow 0 .
$$


Proof. For any $\xi \in M$, let us introduce the following functions defined for $r \in(0,+\infty)$ :

$$
m_{\xi}(r):=\mu\left(\Omega \cap B_{\xi}(r)\right)
$$

and

$$
s_{\xi}(r):=\sigma\left(\partial \Omega \cap B_{\xi}(r)\right) .
$$

STEP 1. We claim that following inequality holds

$$
-\frac{d}{d r}\left(r^{-n} m_{\xi}(r)\right) \leq r^{-n} s_{\xi}(r),
$$

for almost every $r \in\left(0, R_{0}\right)$.

Since the point $\xi$ will be fixed for a while, we skip the subscript $\xi$ from all notation. By Sard's theorem, for almost all $r$, each of the sets $\{x \in M: \rho(x)=r\}$ and $\left\{x \in \partial \Omega:\left.\rho\right|_{\partial \Omega}(x)=r\right\}$ contains no critical points of $\rho$. Therefore, the former set, which is $\partial B(r)$, is a smooth hypersurface on $M$ transversal to $\partial \Omega$.

In other words, the boundary of the set $\Omega_{r}:=\Omega \cap B(r)$,

$$
\partial \Omega_{r}=(\partial \Omega \cap B(r)) \cup(\partial B(r) \cap \Omega),
$$

consists of two hypersurfaces which intersect transversely for almost every $r$.

For every such $r$, let us integrate (2.2) over $\Omega_{r}$, pre-multiplied by $1 / 2$, assuming $r \in\left(0, R_{0}\right)$. We obtain, by using the Green formula, (2.1) and $\rho(x) \leq r$ in $B(r)$ (see Fig. 1)

$$
\begin{aligned}
n \mu\left(\Omega_{r}\right) & \leq \frac{1}{2} \int_{\Omega_{r}} \Delta \rho^{2} d \mu \\
& =\int_{\partial \Omega_{r}} \rho \frac{\partial \rho}{\partial \nu} d \sigma \\
& \leq r \int_{\partial \Omega_{r}}|\nabla \rho| d \sigma \\
& \leq r \sigma\left(\partial \Omega_{r}\right) .
\end{aligned}
$$

Taking into account the splitting (2.9) and definitions (2.6), (2.7) we rewrite (2.10) as follows:

$$
\begin{aligned}
n m(r) & \leq r \sigma(\partial \Omega \cap B(r))+r \sigma(\partial B(r) \cap \Omega) \\
& =r s(r)+r \frac{d m}{d r} .
\end{aligned}
$$




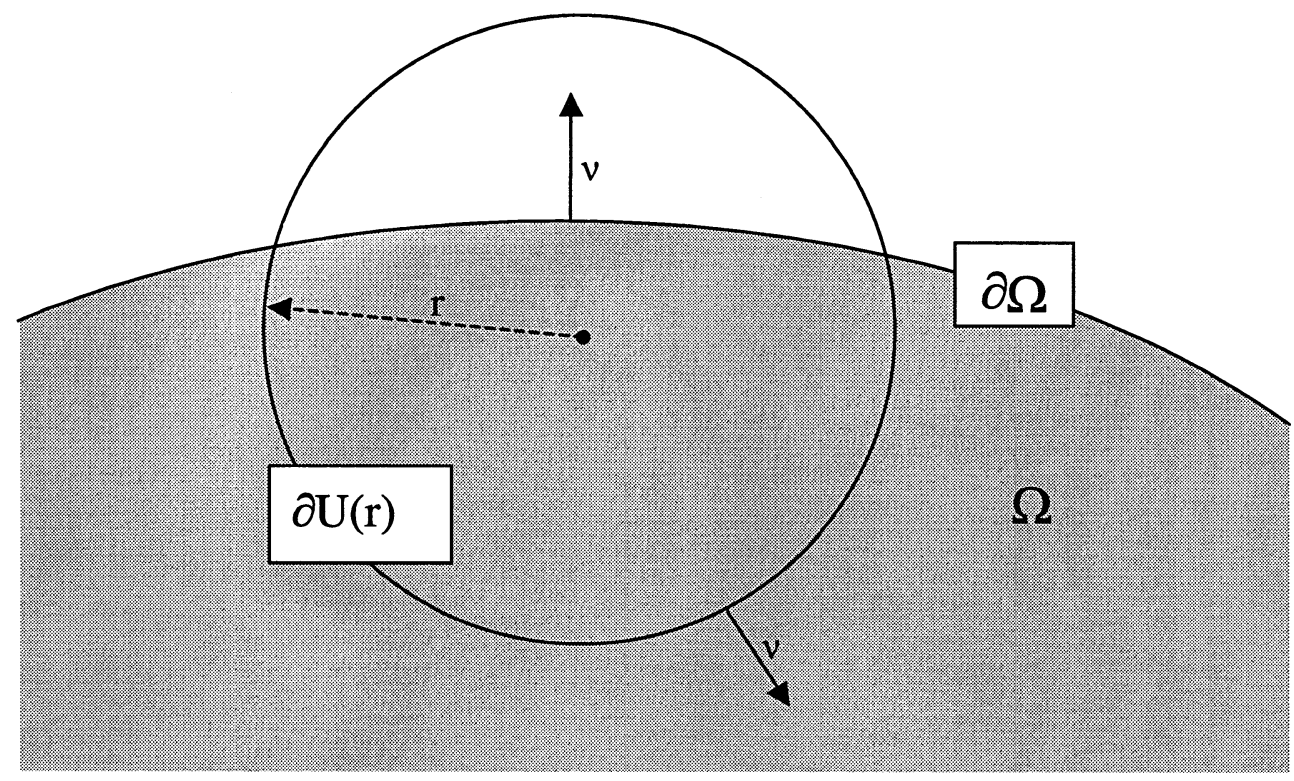

Figure 1: Ball $B(r)$ and set $\Omega$

Finally, dividing (2.11) by $r^{n+1}$, we obtain (2.8).

STEP 2. Let us denote

$$
r_{0}:=\left(\frac{5}{\omega} \mu(\Omega)\right)^{1 / n}
$$

and prove that, for any $\xi \in \Omega$, there exists $r \in\left(0, r_{0}\right)$ such that

$$
s_{\xi}(r) \geq \frac{2^{1-2 n}}{r_{0}} m_{\xi}(4 r) .
$$

We start with the following elementary fact.

Lemma 2.5. Let $f(r)$ be an absolutely continuous positive function on $(0, \infty)$ (see Fig. 2). Assume that, for some $r_{0}>0, C \in(0,4)$ and for almost every $r \in\left(0, r_{0}\right)$, we have

$$
-f^{\prime}(r)<\frac{C}{r_{0}} f(4 r)
$$

Then

$$
\sup _{\left(0, r_{0}\right)} f(r)<\frac{4+3 C}{4-C} \sup _{\left(r_{0}, 4 r_{0}\right)} f(r)
$$




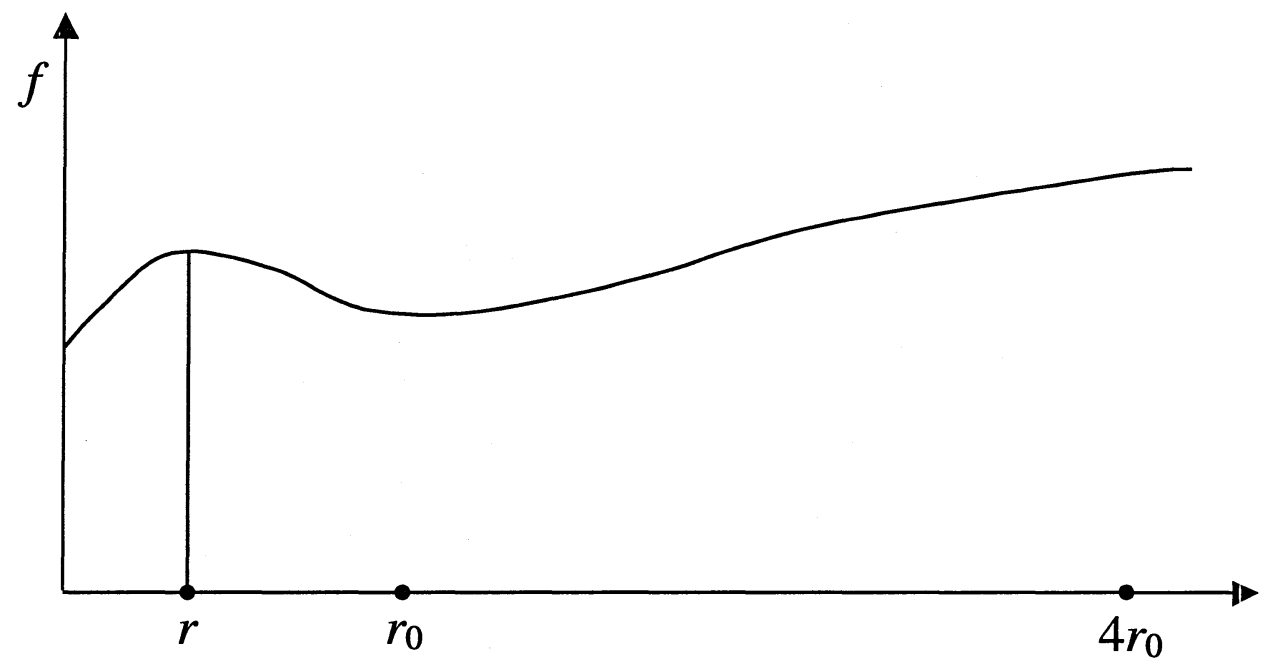

Figure 2: Example of function $f$

Proof. For any $r \in\left(0, r_{0}\right)$, we have, by integrating (2.14),

$$
\begin{aligned}
f(r)-f\left(r_{0}\right) & <\frac{C}{r_{0}} \int_{r}^{r_{0}} f(4 u) d u \\
& \leq \frac{C}{4 r_{0}} \int_{0}^{4 r_{0}} f(t) d t \\
& =\frac{C}{4 r_{0}}\left(\int_{0}^{r_{0}}+\int_{r_{0}}^{4 r_{0}}\right) f(t) d t \\
& \leq \frac{C}{4} \sup _{\left(0, r_{0}\right)} f+\frac{3 C}{4} \sup _{\left(r_{0}, 4 r_{0}\right)} f .
\end{aligned}
$$

By replacing in the left-hand side of $(2.15) f\left(r_{0}\right)$ by $\sup _{\left(r_{0}, 4 r_{0}\right)} f$ and by taking sup over $r \in\left(0, r_{0}\right)$, we obtain from (2.15)-(2.16)

$$
\sup _{\left(0, r_{0}\right)} f-\sup _{\left(r_{0}, 4 r_{0}\right)} f<\frac{C}{4} \sup _{\left(0, r_{0}\right)} f+\frac{3 C}{4} \sup _{\left(r_{0}, 4 r_{0}\right)} f
$$

whence

$$
\left(1-\frac{C}{4}\right) \sup _{\left(0, r_{0}\right)} f<\left(1+\frac{3 C}{4}\right) \sup _{\left(r_{0}, 4 r_{0}\right)} f
$$

which was to be proved. 
Let us now prove (2.13). Assume from the contrary that, for all $r \in$ $\left(0, r_{0}\right)$,

$$
s(r)<\frac{2^{1-2 n}}{r_{0}} m(4 r) .
$$

(where we suppress again the subscript $\xi$ ). Observe that (2.4) and (2.12) imply $r_{0} \leq R_{0}$. Therefore, we can use (2.8) and write

$$
-\frac{d}{d r}\left(r^{-n} m(r)\right)<\frac{2}{r_{0}}(4 r)^{-n} m(4 r)
$$

for almost every $r \in\left(0, r_{0}\right)$.

Next we apply Lemma 2.5, for function $f(r)=r^{-n} m(r)$ and for $C=2$, which yields

$$
\sup _{r \in\left(0, r_{0}\right)} r^{-n} m(r)<5 \sup _{r \in\left(r_{0}, 4 r_{0}\right)} r^{-n} m(r) .
$$

The left-hand side of (2.17) is bounded from below by $\omega$, by the hypothesis (2.3). The right hand-side of (2.17) can be bounded from above by using the fact that $m(r) \leq \mu(\Omega)$, for all $r>0$. Thus, (2.17) implies, together with the definition (2.12) of $r_{0}$,

$$
\omega<\frac{5}{r_{0}^{n}} \mu(\Omega)=\omega
$$

which is a contradiction.

STEP 3. We will prove here that

$$
\sigma(\partial \Omega) \geq \frac{2^{1-2 n}}{r_{0}} \mu(\Omega)
$$

where $r_{0}$ is defined by (2.12). Clearly, (2.18) implies (2.5).

To prove (2.18), let us recall that, for any point $\xi \in \Omega$, there exists $r=r_{\xi} \in\left(0, r_{0}\right)$ such that $(2.13)$ holds. Then, for any $x \in \Omega$, we have, by (2.13),

$$
\sigma\left(\partial \Omega \cap B_{x}\left(r_{x}\right)\right) \geq \frac{2^{1-2 n}}{r_{0}} \mu\left(\Omega \cap B_{x}\left(4 r_{x}\right)\right) .
$$

Let us show that there exists a countable set $T \subset \Omega$ of points $x$ such that all balls $\left\{B_{x}\left(r_{x}\right)\right\}_{x \in T}$ are disjoint whereas the balls $\left\{B_{x}\left(4 r_{x}\right)\right\}_{x \in T}$ cover $\Omega$. If such a family of balls is found already, then we just add up (2.19) for all $x \in T$ and obtain (2.18).

Thus, we are left to prove the following statement. 
Lemma 2.6. (The well-known ball covering argument). Let $(M, d)$ be a metric space with a countable base. Suppose that any point $x$ from a set $\Omega \subset M$ is assigned a metric ball $B_{x}\left(r_{x}\right)$ of radius $r_{x} \in\left(0, r_{0}\right)$. Then there exists a (at most countable) set $S \subset \Omega$ such that all balls $B_{x}\left(r_{x}\right), x \in S$, are disjoint whereas the union of the balls $B_{x}\left(4 r_{x}\right), x \in S$, covers all of the set $\Omega$.

Proof. We construct by induction a sequence of points $x_{i}$ which will form the set $S$, and an auxiliary decreasing sequence of at most countable sets $T_{i} \subset \Omega$. Here $i=0,1,2, \ldots$ is an integer if $\Omega$ is a compact but, in general, $i$ is a transfinite number so that we have to use the transfinite induction.

By the countable basis hypothesis, there exists a countable family of balls $B_{x}\left(r_{x}\right)$ which covers $\Omega$ as well. Let $T_{0}$ be the set of their centers (there will be no point $x_{0}$ ).

Given $i \geq 1$ and assuming that $T_{j}$ has been already constructed for all $j<i$, let us denote

$$
T_{i}^{*}=\bigcap_{j<i} T_{j} .
$$

The inductive process stops at step $i$ if $T_{i}^{*}$ is empty.

Otherwise, choose a point $x_{i} \in T_{i}^{*}$ so that

$$
r_{i}:=r_{x_{i}}>\frac{2}{3} \sup _{x \in T_{i}^{*}} r_{x}
$$

Let us throw away from $T_{i}^{*}$ all points $x$ such that

$$
d\left(x, x_{i}\right) \leq r_{i}+r_{x}
$$

and denote by $T_{i}$ the rest of $T_{i}^{*}$ (see Fig. 3).

Let us prove following two properties of the sequence $\left\{x_{i}\right\}$ :

(i) if $i \neq j$ then the balls $B_{x_{i}}\left(r_{i}\right)$ and $B_{x_{j}}\left(r_{j}\right)$ are disjoint;

(ii) the union of all balls $B_{x_{i}}\left(4 r_{i}\right)$ covers $\Omega$.

Proof of (i). By the construction of $T_{i}$, for any $x \in T_{i}$, the inequality (2.21) fails, which implies that $B_{x_{i}}\left(r_{i}\right)$ and $B_{x}\left(r_{x}\right)$ are disjoint. The same property is true for the previous inductive steps: for any $x \in T_{j}, j<i$, the balls $B_{x_{j}}\left(r_{j}\right)$ and $B_{x}\left(r_{x}\right)$ are disjoint. This can be applied for $x=x_{i}$ because $x_{i} \in T_{i}^{*} \subset T_{j}$, whence (i) follows. 


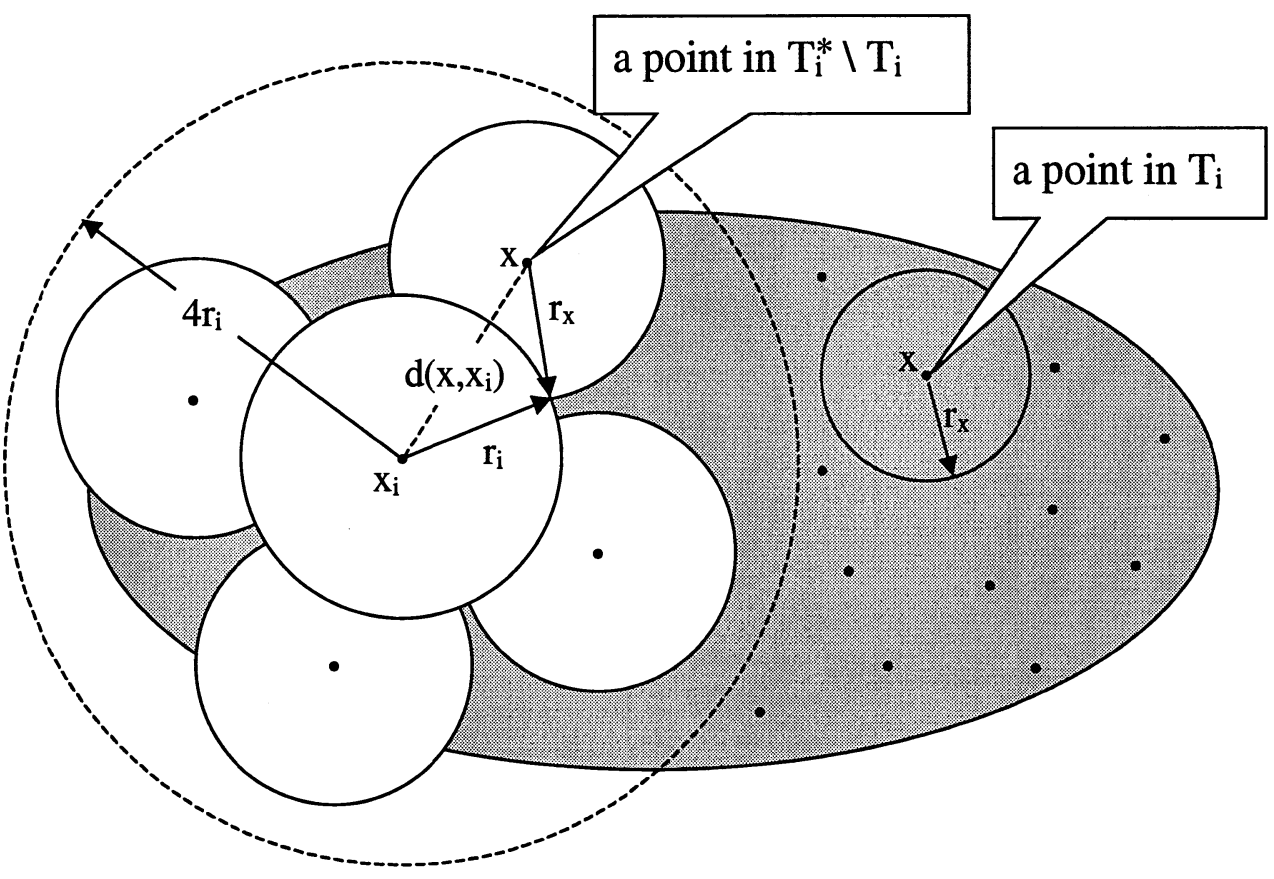

Figure 3: Constructing of $x_{i}$ and $T_{i}$

As a consequence of (i) we see that the points $x_{i}$ and $x_{j}$ are different for different $i$ and $j$. This implies that the inductive process will stop at some $i$ because all $x_{i}$ belong to the countable set $T_{0}$ whereas the set of all transfinite numbers is uncountable.

Proof of (ii). It suffices to show that any ball $B_{x}\left(r_{x}\right)$ centered at $T_{0}$ is covered by one of the balls $B_{x_{i}}\left(4 r_{i}\right)$. For any $x \in T_{0}$, there exists the smallest transfinite number $i$ such that $x \notin T_{i}$ (indeed, as was shown above, for all $i$ large enough, $T_{i}$ is empty whence $x \notin T_{i}$ ). Hence, for any $j<i$, we have $x \in T_{j}$, which implies $x \in T_{i}^{*}$ and $x \in T_{i}^{*} \backslash T_{i}$. By construction, we have (2.21) and $r_{x}<\frac{3}{2} r_{i}$ (the latter follows from (2.20)), whence

$$
d\left(x, x_{i}\right)+r_{x} \leq\left(r_{i}+r_{x}\right)+r_{x}=r_{i}+2 r_{x}<4 r_{i} .
$$

Therefore, $B_{x}\left(r_{x}\right)$ is covered by $B_{x_{i}}\left(4 r_{i}\right)$. 


\section{Eigenvalues of subsets.}

Theorem 3.1. Let us assume that, for any open precompact set $\Omega \subset M$ with smooth boundary such that

$$
\mu(\Omega) \leq v_{0},
$$

the following inequality holds

$$
\sigma(\partial \Omega) \geq c \mu(\Omega)^{1-1 / n},
$$

where $c>0, v_{0}>0$ and $n>1$ are constants. Then, for any open precompact open set $\Omega \subset M$ and for any integer $k$ such that

$$
k \geq \frac{(n+2) e}{v_{0}} \mu(\Omega),
$$

we have

$$
\lambda_{k}(\Omega) \geq a\left(\frac{k}{\mu(\Omega)}\right)^{2 / n},
$$

where $a=e^{-2 / n} 2^{-1}(n+2)^{1-2 / n} c^{2}$.

Proof. The proof consists of four steps.

STEP 1. We first prove Theorem 3.1 for $k=1$. We replace hypothesis (3.2) by a weaker assumption $\mu(\Omega) \leq v_{0}$, which suffices in this case. The following argument is due to Cheeger [6] and Maz'ya [23].

Given a non-negative function $f \in C_{0}^{\infty}(\Omega)$, we denote

$$
\Omega_{t}=\{x: f(x)>t\} .
$$

Since $\mu\left(\Omega_{t}\right) \leq v_{0}$ and, by Sard's theorem, the boundary $\partial \Omega_{t}$ is smooth, for almost all $t$, we may apply the isoperimetric inequality (3.1) for $\Omega_{t}$ and obtain

$$
\sigma\left(\partial \Omega_{t}\right) \geq c \mu\left(\Omega_{t}\right)^{1-1 / n},
$$

for almost all $t$.

Next, we use the co-area formula

$$
\int_{M}|\nabla f| d \mu=\int_{0}^{\infty} \sigma\left(\partial \Omega_{t}\right) d t
$$


which implies with (3.4)

$$
\begin{aligned}
\int_{M}|\nabla f| d \mu & \geq c \int_{0}^{\infty} \mu\left(\Omega_{t}\right)^{1-1 / n} d t \\
& \geq \frac{c}{\mu(\Omega)^{1 / n}} \int_{0}^{\infty} \mu\left(\Omega_{t}\right) d t \\
& =\frac{c}{\mu(\Omega)^{1 / n}} \int_{M} f d \mu,
\end{aligned}
$$

and

$$
\int_{M} f d \mu \leq c^{-1} \mu(\Omega)^{1 / n} \int_{M}|\nabla f| d \mu .
$$

We have, by the Cauchy-Schwarz inequality,

$$
\int_{M}\left|\nabla f^{2}\right| d \mu=2 \int_{M} f|\nabla f| \leq 2\left[\int_{M} f^{2} d \mu \int_{M}|\nabla f|^{2} d \mu\right]^{1 / 2}
$$

By applying (3.6) to $f^{2}$ instead of $f$ and by (3.7), we obtain

$$
\begin{aligned}
\int_{M} f^{2} d \mu & \leq c^{-1} \mu(\Omega)^{1 / n} \int_{M}\left|\nabla f^{2}\right| d \mu \\
& \leq 2 c^{-1} \mu(\Omega)^{1 / n}\left[\int_{M} f^{2} d \mu \int_{M}|\nabla f|^{2} d \mu\right]^{1 / 2}
\end{aligned}
$$

whence

$$
\int_{M} f^{2} d \mu \leq 4 c^{-2} \mu(\Omega)^{2 / n} \int_{M}|\nabla f|^{2} d \mu
$$

and

$$
\lambda_{1}(\Omega)=\inf _{\substack{f \in C_{0}^{\infty}(\Omega) \\ f \neq 0}} \frac{\int_{M}|\nabla f|^{2} d \mu}{\int_{M} f^{2} d \mu} \geq \frac{c^{2}}{4} \mu(\Omega)^{-2 / n} .
$$

STEP 2. Let us prove the following lemma which is a localized version of similar statements in [17] and [15].

Lemma 3.2. Assume that, for any open precompact set $\Omega \subset M$ such that

$$
\mu(\Omega) \leq v_{0},
$$


the following inequality holds

$$
\lambda_{1}(\Omega) \geq \Lambda(\mu(\Omega))
$$

where $\Lambda(\cdot)$ is a non-negative non-increasing function on $(0, \infty)$. Let $u(x)$ be a Lipschitz non-negative function on $M$ with compact support and let us denote

$$
\int_{M} u d \mu=\alpha \quad \text { and } \quad \int_{M} u^{2} d \mu=\beta .
$$

Then, for any $s \geq s_{0}:=\alpha / v_{0}$,

$$
\int_{M}|\nabla u|^{2} d \mu \geq(\beta-2 s \alpha) \Lambda\left(s^{-1} \alpha\right)
$$

Proof. We start with the obvious inequality

$$
u^{2} \leq(u-s)_{+}^{2}+2 s u
$$

which holds for any $s \geq 0$ and which implies

$$
\beta=\int_{M} u^{2} d \mu \leq \int_{\{u>s\}}(u-s)^{2} d \mu+2 s \int_{M} u d \mu
$$

and

$$
\beta-2 s \alpha \leq \int_{\{u>s\}}(u-s)^{2} d \mu
$$

On the other hand,

$$
\mu\{u>s\} \leq s^{-1} \alpha .
$$

Therefore, if $s \geq s_{0}:=\frac{\alpha}{v_{0}}$, then the set $\Omega_{s}:=\{u>s\}$ (see Fig. 4) satisfies the hypothesis (3.9), whence

$$
\lambda_{1}\left(\Omega_{s}\right) \geq \Lambda\left(\mu\left(\Omega_{s}\right)\right) \geq \Lambda\left(s^{-1} \alpha\right) .
$$

This implies

$$
\Lambda\left(s^{-1} \alpha\right) \int_{\Omega_{s}}(u-s)^{2} d \mu \leq \int_{\Omega_{s}}|\nabla u|^{2} d \mu .
$$

Comparison of (3.11) and (3.12) yields (3.10). 


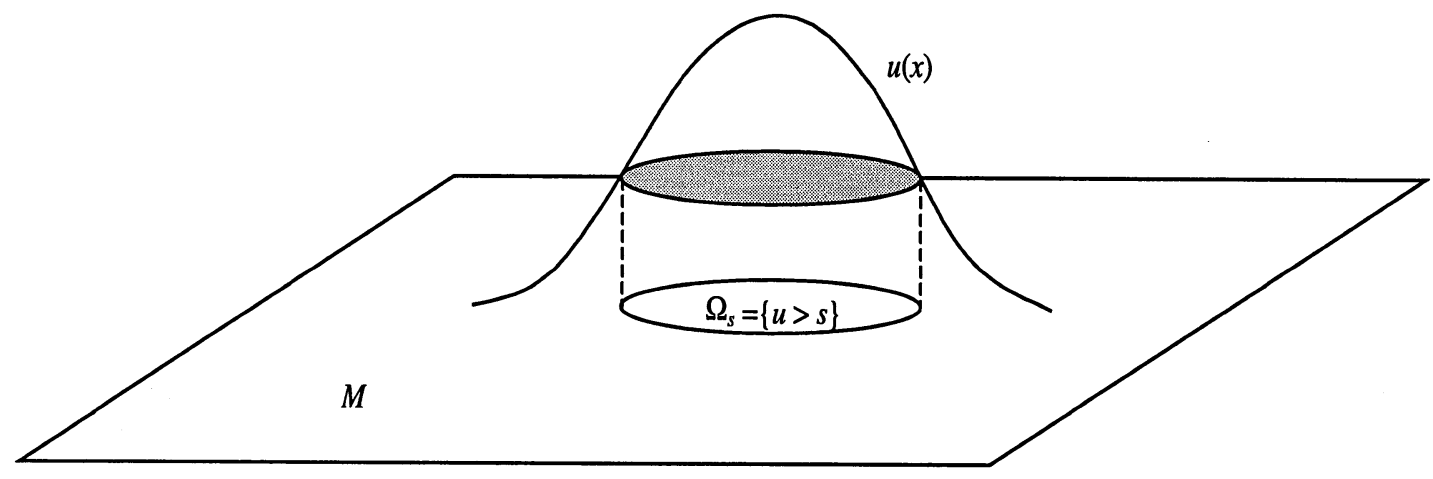

Figure 4: Function $u$ and sets $\Omega_{s}$

We apply Lemma 3.2 for $\Lambda(v)=\frac{c^{2}}{4} v^{-2 / n}$ and for a function $u$ such that $\int_{M} u d \mu \leq 1$, that is $\alpha \leq 1$. For this function, (3.10) becomes

$$
\int_{M}|\nabla u|^{2} d \mu \geq \frac{c^{2}}{4} s^{2 / n}(\beta-2 s)
$$

provided $s \geq v_{0}^{-1}$.

The right-hand side of (3.13) takes the maximum at $s=\frac{\beta}{n+2}$. Let us choose the optimal $s$. If

$$
\frac{\beta}{n+2} \geq v_{0}^{-1}
$$

then we let $s=\frac{\beta}{n+2}$ and obtain

$$
\int_{M}|\nabla u|^{2} d \mu \geq \frac{c^{2}}{4} \frac{n}{(n+2)^{1+2 / n}} \beta^{1+2 / n}
$$

If

$$
\frac{\beta}{n+2}<v_{0}^{-1}
$$

then the best value of $s$ in (3.13) is $v_{0}^{-1}$, whence

$$
\int_{M}|\nabla u|^{2} d \mu \geq \frac{c^{2}}{4} v_{0}^{-2 / n}\left(\beta-2 v_{0}^{-1}\right)_{+} .
$$




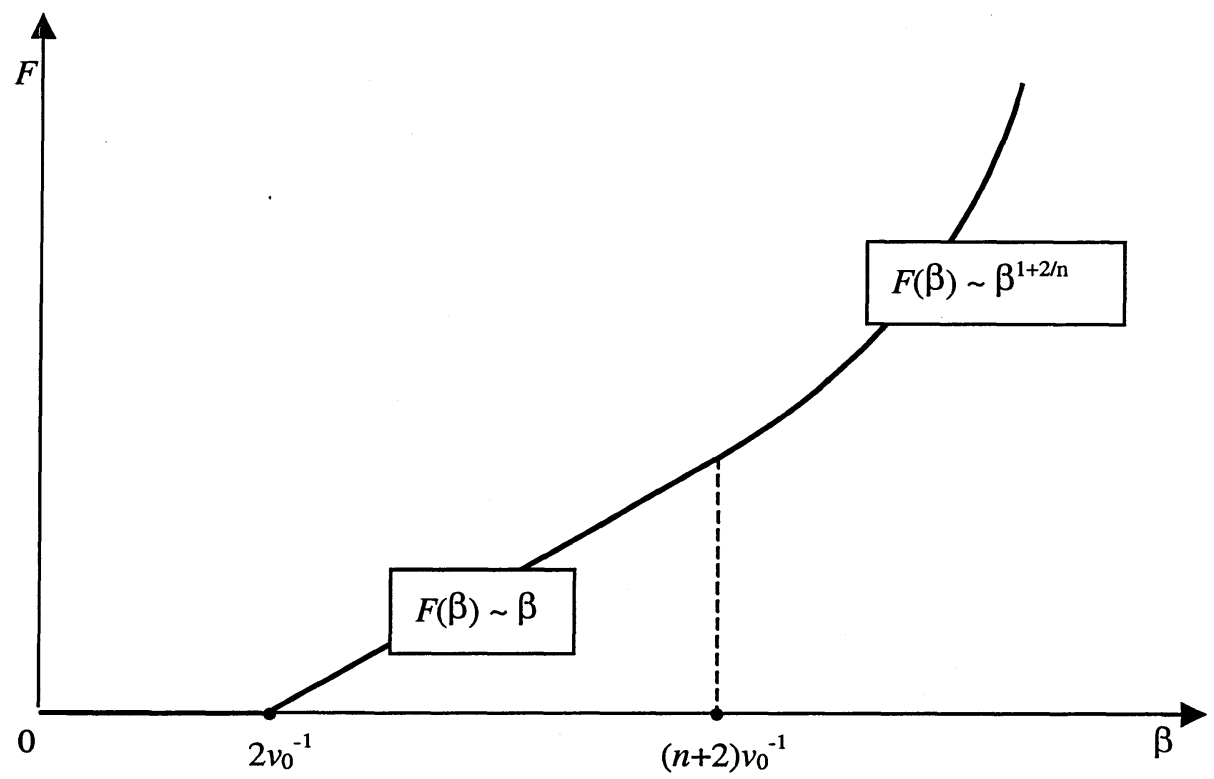

Figure 5: Function $F(\beta)$

We summarize the above inequalities in the following one:

$$
\int_{M}|\nabla u|^{2} d \mu \geq F(\beta):=\frac{c^{2}}{4} \begin{cases}0, & \beta \leq 2 v_{0}^{-1} \\ v_{0}^{-2 / n}\left(\beta-2 v_{0}^{-1}\right), & 2 v_{0}^{-1}<\beta \leq(n+2) v_{0}^{-1}, \\ \frac{n}{(n+2)^{1+2 / n}} \beta^{1+2 / n}, & \beta>(n+2) v_{0}^{-1},\end{cases}
$$

(see Fig. 5). In fact, we will use this inequality only in the range $\beta>$ $(n+2) v_{0}^{-1}$. In this range, (3.14) is a Nash type inequality. Nash [26] used a similar inequality to obtain upper bounds of the heat kernel. So we do in the next step.

STEP 3. For any precompact region $\Omega \subset M$, we denote by $p_{\Omega}(t, x, y)$ the Dirichlet heat kernel in $\Omega$; that is, the kernel of the minimal heat semigroup $e^{t \Delta}$ in $\Omega$ with respect to the measure $\mu$ (see [4], [7], [11], [16] for detailed definition and properties of heat kernels).

Let us prove that, for any $\Omega$, the heat kernel admits the following upper bound, for all $t>0$ and $x \in \Omega$,

$$
p_{\Omega}(t, x, x) \leq \max \left(K_{0}, K t^{-n / 2}\right),
$$


where

$$
K_{0}=(n+2) v_{0}^{-1} \text { and } K=c^{-n} 2^{n / 2}(n+2)^{n / 2+1} \text {. }
$$

Fix $x \in \Omega$ and denote

$$
I(t)=\int_{\Omega} p_{\Omega}^{2}(t, x, y) d \mu(y) .
$$

By the semigroup property and by the symmetry of the heat kernel, we have $I(t)=p_{\Omega}(2 t, x, x)$. Denote also $u(t, y)=p_{\Omega}(t, x, y)$. The heat equation

$$
u_{t}=\Delta u
$$

implies, by multiplying by $u$ and by integrating over $\Omega$, that

$$
I^{\prime}(t)=-2 \int_{\Omega}|\nabla u|^{2} d \mu(y) .
$$

Since $\int_{\Omega} u(t, y) d \mu(y) \leq 1$, we can apply (3.14), which yields

$$
I^{\prime}(t) \leq-2 F(I) \text {. }
$$

Function $I(t)$ is decreasing in $t$, and $I(t) \rightarrow \infty$ as $t \downarrow 0$. We would like to integrate (3.17) when $I(t)$ stays in the range $\left((n+2) v_{0}^{-1}, \infty\right)$. This means that the variable $t$ should vary within $\left(0, t_{0}\right)$, where $I\left(t_{0}\right)=(n+2) v_{0}^{-1}$. By integrating (3.17) from 0 to $t<t_{0}$, we obtain

$$
\int_{I}^{\infty} \frac{d I}{F(I)} \geq 2 t
$$

and (see Fig. 6)

$$
I(t) \leq c^{-n}(n+2)^{n / 2+1} t^{-n / 2} .
$$

Thus, if $I(t) \geq(n+2) v_{0}^{-1}$, then (3.18) holds. Otherwise, $I(t)<(n+$ 2) $v_{0}^{-1}$, and we can write, for all $t>0$,

$$
I(t) \leq \max \left((n+2) v_{0}^{-1}, c^{-n}(n+2)^{n / 2+1} t^{-n / 2}\right)
$$

and, changing $t$ to $t / 2$

$$
p_{\Omega}(t, x, x) \leq \max \left((n+2) v_{0}^{-1}, c^{-n} 2^{n / 2}(n+2)^{n / 2+1} t^{-n / 2}\right) .
$$


$990 \quad$ Fang Chung, Alexander Grigor'yan and Shing-Tung Yau

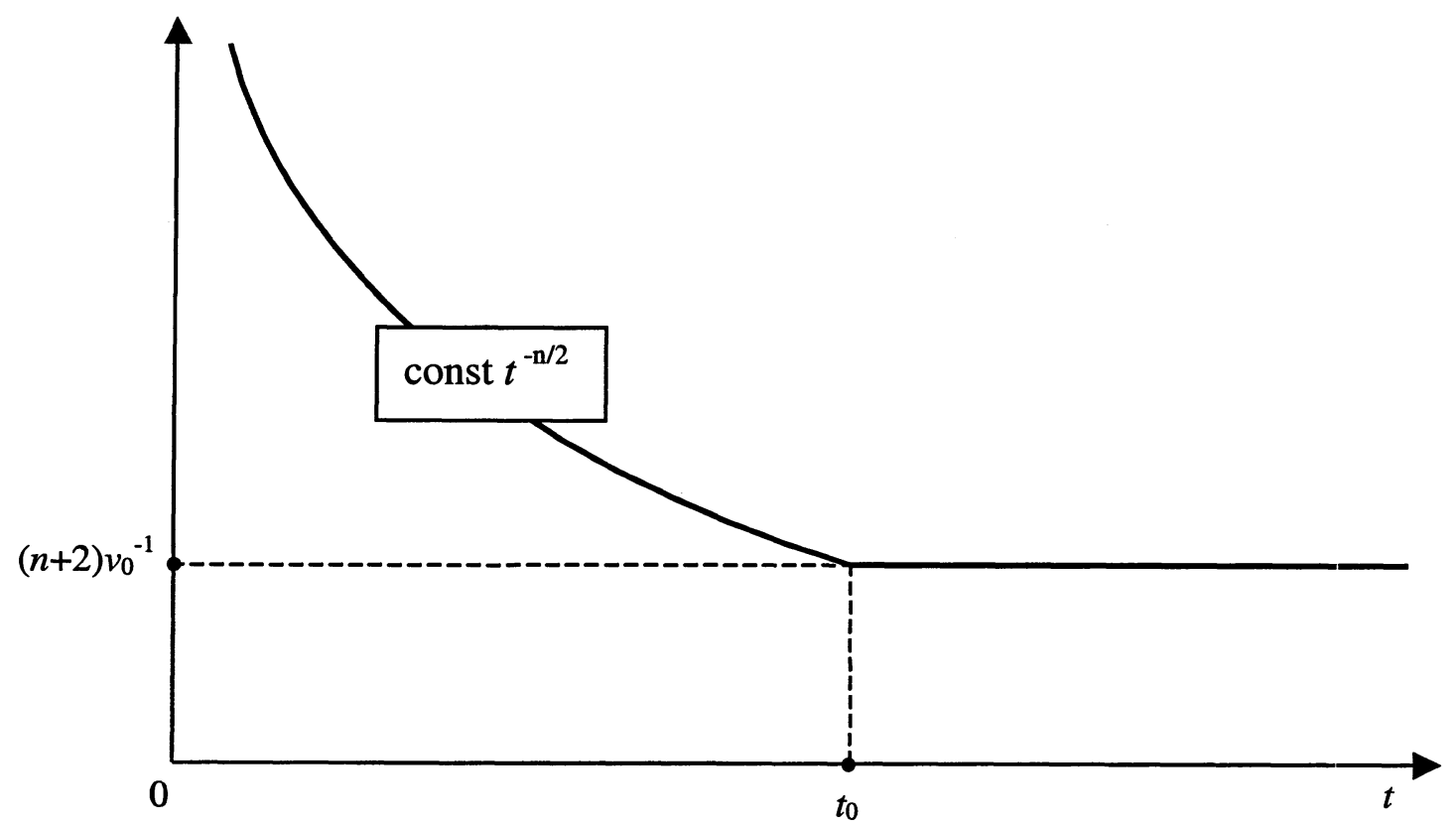

Figure 6: Upper bound for $I(t)$

STEP 4. Let us finally prove (3.3), assuming (3.2). What follows is a modification of the argument of Cheng and $\mathrm{Li}[8]$.

Inequality (3.15) implies

$$
\int_{\Omega} p_{\Omega}(t, x, x) d \mu(x) \leq \mu(\Omega) \max \left(K_{0}, K t^{-n / 2}\right) .
$$

By the trace formula, we have, for any $k \geq 1$,

$$
\int_{\Omega} p_{\Omega}(t, x, x) d \mu(x)=\sum_{j=1}^{\infty} e^{-\lambda_{j}(\Omega) t} \geq k e^{-\lambda_{k}(\Omega) t}
$$

whence, by (3.19), we obtain

$$
k e^{-\lambda_{k}(\Omega) t} \leq \mu(\Omega) \max \left(K_{0}, K t^{-n / 2}\right)
$$

and

$$
\lambda_{k}(\Omega) \geq \frac{1}{t} \log \frac{k}{\mu(\Omega) \max \left(K_{0}, K t^{-n / 2}\right)} .
$$




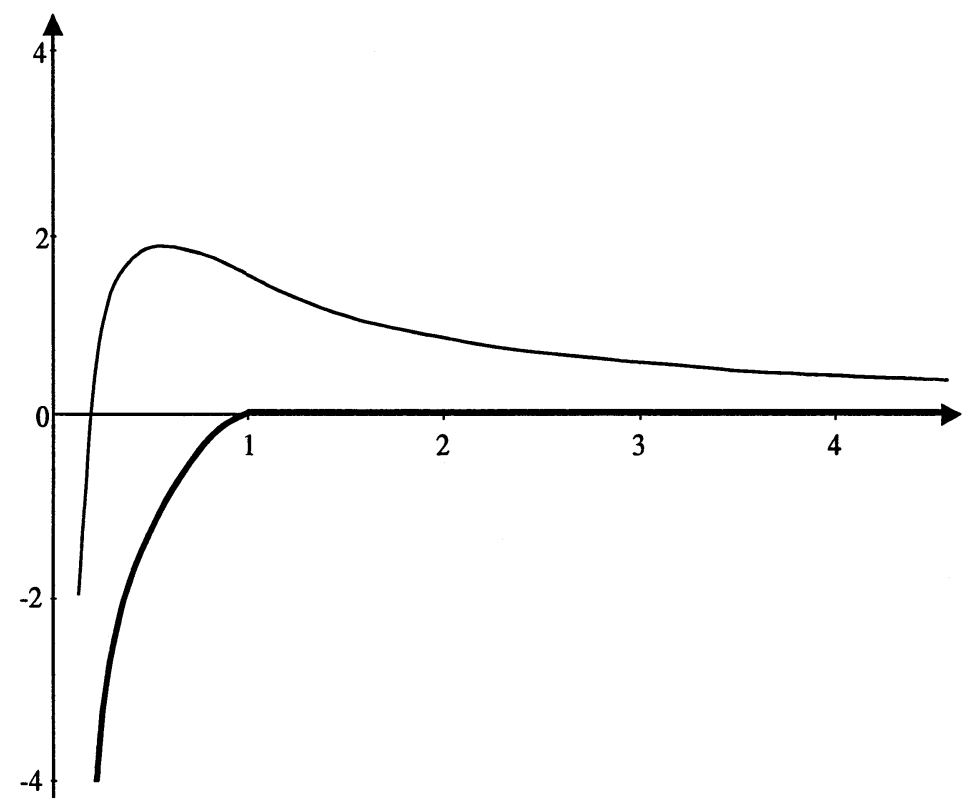

Figure 7: Functions $t \rightarrow \frac{1}{t} \log \frac{5}{\max \left(1, t^{-1}\right)} \quad$ (thin) and $t \quad \rightarrow$ $\frac{1}{t} \log \frac{1}{\max \left(1, t^{-1}\right)}($ thick $)$

Let us choose here $t$ to satisfy

$$
\frac{k}{\mu(\Omega) K t^{-n / 2}}=e
$$

or

$$
t=\left(\frac{e K \mu(\Omega)}{k}\right)^{2 / n}
$$

For this $t$, we claim that

$$
K_{0} \leq K t^{-n / 2}
$$

Indeed, by (3.21) and (3.16), this is equivalent to

$$
\mu(\Omega) \leq \frac{k}{K_{0} e}=\frac{v_{0}}{(n+2) e} k
$$

which is true by the hypothesis (3.2).

Let us mention that if $k$ is not big enough then the right hand side of (3.20) may be non-positive for all $t$. In this case, we get no non-trivial lower 
bound for $\lambda_{k}$. Typical graphs of the right-hand side of (3.20) are shown on Fig. 7.

Hence, (3.20) implies, for $t$ from (3.21),

$$
\lambda_{k}(\Omega) \geq\left(\frac{e K \mu(\Omega)}{k}\right)^{-2 / n}=e^{-2 / n} 2^{-1}(n+2)^{1-2 / n} c^{2}\left(\frac{k}{\mu(\Omega)}\right)^{2 / n},
$$

which was to be proved.

By putting together Theorem 2.1 and 3.1, we obtain the following Corollary.

Corollary 3.3. Under the hypotheses of Theorem 2.1, for any precompact open set $\Omega \subset M$ and for any integer $k \geq 1$ such that

$$
k \geq \kappa \frac{\mu(\Omega)}{\omega R_{0}^{n}}
$$

where $\kappa=\kappa(n)$, we have

$$
\lambda_{k}(\Omega) \geq a\left(\frac{k}{\mu(\Omega)}\right)^{2 / n}
$$

where $a=c_{1}(n) \omega^{2 / n}>0$.

Indeed, by Theorem 2.1, we have the isoperimetric inequality

$$
\sigma(\partial \Omega) \geq c \mu(\Omega)^{1-1 / n},
$$

where $c=2^{1-2 n}\left(\frac{\omega}{5}\right)^{1 / n}$, provided

$$
\mu(\Omega) \leq v_{0}:=\frac{1}{5} \omega R_{0}^{n} .
$$

We feed this to Theorem 3.1 and obtain

$$
\lambda_{k}(M) \geq c_{1}(n) \omega^{2 / n}\left(\frac{k}{\mu(\Omega)}\right)^{2 / n},
$$

provided

$$
k \geq \frac{5 e(n+2)}{\omega R_{0}^{n}} \mu(\Omega) .
$$

Theorem 1.1 from Introduction is a particular case of Theorem 2.1 and Corollary 3.3, for $n=\operatorname{dim} M$ and for $\mu$ being the Riemannian measure. The hypothesis (2.3) of Theorem 2.1 holds automatically because $\mu\left(B_{\xi}(R)\right) \sim$ $\omega_{n} R^{n}$ as $R \rightarrow 0$. 


\section{Sobolev inequality.}

Let us introduce the notation

$$
\|f\|_{\alpha}=\left[\int_{M}|f|^{\alpha} d \mu\right]^{1 / \alpha}
$$

Theorem 4.1. Assume that, for any precompact open set $\Omega \subset M$ with smooth boundary, such that

$$
\mu(\Omega) \leq v_{0}
$$

the following inequality holds

$$
\sigma(\partial \Omega) \geq c \mu(\Omega)^{1-1 / n},
$$

where $c>0, v_{0}>0$ and $n>1$. Then, for any Lipschitz function $f$ with compact support, the following inequality holds

$$
\int_{M}|\nabla f| d \mu+c \Theta\left(\Omega_{0}\right) \int_{M}|f| d \mu \geq c 2^{-1 / n}\left[\int_{M}|f|^{\frac{n}{n-1}} d \mu\right]^{\frac{n-1}{n}}
$$

where $\Omega_{0}=\{x \in M:|f(x)|>0\}$ and

$$
\Theta(\Omega):= \begin{cases}0, & \text { if } \mu(\Omega) \leq v_{0} \\ v_{0}^{-1} \mu(\Omega)^{1-1 / n}, & \text { if } \mu(\Omega)>v_{0} .\end{cases}
$$

Furthermore, for any $p \in[1, n)$, we have

$$
C\|\nabla f\|_{p}+c \Theta\left(\Omega_{0}\right)\|f\|_{p} \geq c 2^{1 / p-1-1 / n}\|f\|_{\frac{n p}{n-p}}
$$

with some constant $C=C(n, p)>0$.

Remark 4.2. In inequality (4.2), one can get rid of the term $2^{-1 / n}$ provided $\mu\left(\Omega_{0}\right) \leq v_{0}$. In this case, we get the inequality

$$
\int_{M}|\nabla f| d \mu \geq c\left[\int_{M}|f|^{\frac{n}{n-1}} d \mu\right]^{\frac{n-1}{n}}
$$

where the constant $c$ is sharp - see [14], [23], [18]. 


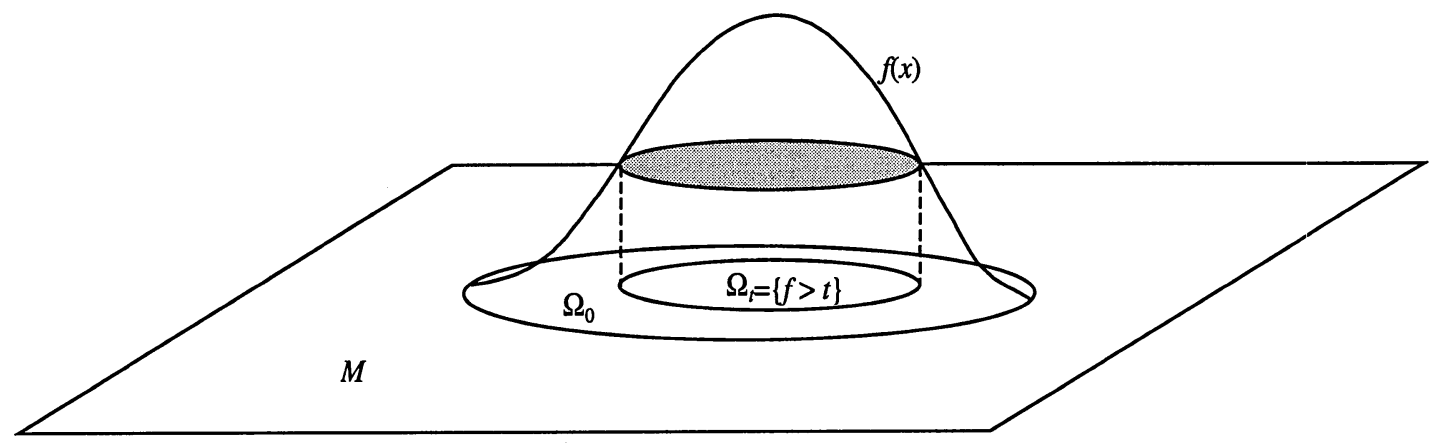

Figure 8: Level sets of function $f(x)$

Proof. Let us first prove (4.2). Without loss of generality, we may assume $f \in C_{0}^{\infty}(M)$ and $f \geq 0$. Denote $\Omega_{t}:=\{x: f(x)>t\}$ (see Fig. 8).

Let $t_{0}$ be large enough so that

$$
\mu\left(\Omega_{t_{0}}\right) \leq v_{0}
$$

(such $t_{0}$ always exists). Then, for any $t \geq t_{0}$, we have also $\mu\left(\Omega_{t}\right) \leq v_{0}$ and, by hypotheses, the isoperimetric inequality (4.1) holds for $\Omega_{t}$, that is

$$
\sigma\left(\partial \Omega_{t}\right) \geq c \mu\left(\Omega_{t}\right)^{1-1 / n} .
$$

The co-area formula (3.5) implies

$$
\int_{M}|\nabla f| d \mu \geq \int_{t_{0}}^{\infty} \sigma\left(\partial \Omega_{t}\right) d t
$$

and

$$
\int_{M}|\nabla f| d \mu \geq c \int_{t_{0}}^{\infty} \mu\left(\Omega_{t}\right)^{1-1 / n} d t=c \int_{0}^{\infty} \mu\left(\Omega_{t_{0}+s}\right)^{\frac{n-1}{n}} d s .
$$

Let us use the inequality of Hardy, Littlewood and Pólya which says that, for any non-negative non-increasing function $h$ on $(0, \infty)$ and for any $\alpha \geq 1$,

$$
\int_{0}^{\infty} h^{1 / \alpha}(s) d s \geq\left[\int_{0}^{\infty} h(s) d\left(s^{\alpha}\right)\right]^{1 / \alpha} .
$$

Applying it to $h(s)=\mu\left(\Omega_{t_{0}+s}\right)$ and

$$
\alpha=\frac{n}{n-1}
$$


we obtain

$$
\begin{aligned}
\int_{0}^{\infty} \mu\left(\Omega_{t_{0}+s}\right)^{1 / \alpha} d s & \geq\left[\int_{0}^{\infty} \mu\left(\Omega_{t_{0}+s}\right) d\left(s^{\alpha}\right)\right]^{1 / \alpha} \\
& =\left[\int_{t_{0}}^{\infty} \mu\left(\Omega_{t}\right) d\left(\left(t-t_{0}\right)^{\alpha}\right)\right]^{1 / \alpha} \\
& =\left[\int_{M}\left(f-t_{0}\right)_{+}^{\alpha} d \mu\right]^{1 / \alpha} .
\end{aligned}
$$

If $\mu\left(\Omega_{0}\right) \leq v_{0}$ then we can take $t_{0}=0$ and obtain from (4.6)-(4.7) the sharp Sobolev inequality (4.4). This is the classical way of proving the Sobolev inequality, which is due to Federer - Fleming [14] and Maz'ya [22], [23, Section 2.3.3].

In general, $t_{0}$ may be positive. We apply the elementary inequality

$$
(x+y)^{a} \leq 2^{\alpha-1}\left(x^{\alpha}+y^{\alpha}\right),
$$

which holds for all $\alpha \geq 1$ and positive $x, y$, and obtain

$$
\left(f-t_{0}\right)_{+}^{\alpha} \geq 2^{1-\alpha} f^{\alpha}-t_{0}^{\alpha},
$$

whence

$$
\int_{M}\left(f-t_{0}\right)_{+}^{\alpha} d \mu=\int_{\Omega_{0}}\left(f-t_{0}\right)_{+}^{\alpha} d \mu \geq 2^{1-\alpha} \int_{\Omega_{0}} f^{\alpha} d \mu-t_{0}^{\alpha} \mu\left(\Omega_{0}\right) .
$$

By another elementary inequality

$$
(x-y)_{+}^{1 / \alpha} \geq x^{1 / \alpha}-y^{1 / \alpha}
$$

we have

$$
\left[2^{1-\alpha} \int_{\Omega_{0}} f^{\alpha} d \mu-t_{0}^{\alpha} \mu\left(\Omega_{0}\right)\right]_{+}^{\frac{1}{\alpha}} \geq 2^{\frac{1}{\alpha-1}}\left[\int_{\Omega_{0}} f^{\alpha} d \mu\right]^{\frac{1}{\alpha}}-t_{0} \mu\left(\Omega_{0}\right)^{\frac{1}{\alpha}} .
$$

Combining together (4.6)-(4.9), we obtain

$$
\int_{M}|\nabla f| d \mu \geq c 2^{-1 / n}\left[\int_{M} f^{\frac{n}{n-1}} d \mu\right]^{\frac{n-1}{n}}-c t_{0} \mu\left(\Omega_{0}\right)^{\frac{n-1}{n}} .
$$

Let us choose $t_{0}$ now to satisfy (4.5). If $\mu\left(\Omega_{0}\right) \leq v_{0}$ then we take $t_{0}=0$ and obtain (4.4). If $\mu\left(\Omega_{0}\right)>v_{0}$, then we observe that

$$
\mu\left(\Omega_{t_{0}}\right) \leq \frac{1}{t_{0}} \int_{M} f d \mu .
$$


Therefore, (4.5) is satisfied for

$$
t_{0}=\frac{1}{v_{0}} \int_{M} f d \mu .
$$

For this $t_{0}$, we deduce form (4.10)

$$
\int_{M}|\nabla f| d \mu+c v_{0}^{-1} \mu\left(\Omega_{0}\right)^{1-1 / n} \int_{M} f d \mu \geq c 2^{-1 / n}\left[\int_{M} f^{\frac{n}{n-1}} d \mu\right]^{\frac{n-1}{n}}
$$

which was to be proved.

Now we prove (4.3). Denote $g:=\left(f-t_{0}\right)_{+}$and $G:=\{g>0\}=\Omega_{t_{0}}$. By the choice of $t_{0}$, we have $\mu(G) \leq v_{0}$. Therefore, as was shown above, the function $g$ satisfies the Sobolev inequality (4.4). Moreover, (4.4) holds as well for the function $g^{\kappa}$, for any $\kappa \geq 1$. Applying also the Hölder inequality, we obtain

$$
\begin{aligned}
c\left[\int g^{\frac{\kappa n}{n-1}} d \mu\right]^{\frac{n-1}{n}} & \leq \kappa \int g^{\kappa-1}|\nabla g| d \mu \\
& \leq \kappa\left[\int|\nabla g|^{p} d \mu\right]^{1 / p}\left[\int g^{(\kappa-1) q} d \mu\right]^{1 / q}
\end{aligned}
$$

where $q$ is defined by $\frac{1}{p}+\frac{1}{q}=1$. Choose $\kappa=\frac{p(n-1)}{n-p}$ so that

$$
\frac{\kappa n}{n-1}=(\kappa-1) q \text {. }
$$

Then after obvious simplifications, (4.12) implies

$$
c\|g\|_{\frac{n p}{n-p}} \leq C\|\nabla g\|_{p}
$$

where $C=C(n, p)$.

By setting $\alpha=\frac{n p}{n-p}$ we can rewrite this as

$$
C\|\nabla f\|_{p} \geq c\left\|\left(f-t_{0}\right)_{+}\right\|_{\alpha} .
$$

We apply again inequalities (4.8), (4.9) and obtain

$$
C\|\nabla f\|_{p} \geq c 2^{1 / \alpha-1}\|f\|_{\alpha}-c t_{0} \mu\left(\Omega_{0}\right)^{1 / \alpha} .
$$

Taking $t_{0}$ as in (4.11), we have 


$$
C\|\nabla f\|_{p}+c \frac{\mu\left(\Omega_{0}\right)^{1 / \alpha}}{v_{0}}\|f\|_{1} \geq c 2^{1 / \alpha-1}\|f\|_{\alpha} .
$$

Finally, we observe that, by the Cauchy-Schwarz inequality,

$$
\|f\|_{1}=\int_{\Omega_{0}} f d \mu \leq \mu\left(\Omega_{0}\right)^{1-1 / p}\|f\|_{p}
$$

whence

$$
C\|\nabla f\|_{p}+c \frac{\mu\left(\Omega_{0}\right)^{1-1 / n}}{v_{0}}\|f\|_{p} \geq c 2^{1 / \alpha-1}\|f\|_{\alpha}
$$

which was to be proved.

\section{Analysis on weighted graphs.}

Let $\Gamma$ denote a connected graph with the vertex set $V$ and the edge set $E$. For vertices $x, y \in V$, we write $x \sim y$ if $x$ and $y$ are neighbors in $\Gamma$, that is, $x$ and $y$ are adjacent and joined by an edge, which will be denoted by $x y$. Here we consider locally finite graphs i. e., every vertex has a finite number of neighbors.

For a subset $\Omega$ of vertices in $\Gamma$, we denote by $\partial \Omega$ the set of edges which join a vertex in $\Omega$ with a vertex outside $\Omega$. We normally assume that each edge in $\partial \Omega$ is oriented so that it points outwards from $\Omega$ unless otherwise specified.

\subsection{Measures on graphs.}

We consider a weighted graph in which each edge $e$ is associated with a positive edge weight $\sigma_{e}$. For any edge set $S \subset E$, we define its measure by

$$
\sigma(S)=\sum_{e \in S} \sigma_{e}
$$

If edge $e$ connects vertices $x, y$ then we write also $\sigma_{e}=\sigma_{x y}=\sigma_{y x}$. Extend the function $\sigma_{x y}$ by zero for those $x$ and $y$ which are not neighbors so that it becomes a (symmetric) function on $V \times V$. Depending on the context, we may regard $\sigma_{x y}$ as a measure on edges or as a function on pairs of vertices. 
For any vertex $x \in V$, we introduce the vertex weight

$$
\mu_{x}:=\sum_{\{y: y \sim x\}} \sigma_{x y} .
$$

For example, if $\sigma_{e}=1$ on all edges then $\mu_{x}$ is the number of neighbors of the vertex $x$, that is, the degree of $x$. We regard $\mu_{x}$ as a measure on vertices. Namely, for any subset $\Omega$ of vertices, we define

$$
\mu(\Omega)=\sum_{x \in \Omega} \mu_{x} .
$$

A graph $\Gamma$ equipped with the weight $\sigma$ (and its derivative - measure $\mu$ ) is called a weighted graph and is denoted by $(\Gamma, \sigma)$.

\subsection{Discrete Laplacian.}

Given a real-valued function $f$ on the vertex set of $(\Gamma, \sigma)$, we consider its gradient $\nabla$ and the Laplace operator $\Delta$. The gradient of $f$ assigns the following value to each ordered pair $x, y \in V$

$$
\nabla_{x y} f=f(y)-f(x) .
$$

Expression $\nabla f$ can also be considered as a function on oriented edges: if $e=\overrightarrow{x y}$ then $\nabla_{e} f=\nabla_{x y} f$.

The Laplace operator $\Delta$ is defined on all functions $f$ on $V$ as follows:

$$
\Delta f(x)=\frac{1}{\mu_{x}} \sum_{\{y: y \sim x\}}\left(\nabla_{x y} f\right) \sigma_{x y}=\frac{1}{\mu_{x}} \sum_{\{y: y \sim x\}} f(y) \sigma_{x y}-f(x) .
$$

The matrix of $\Delta$ has in the bases $\left\{\delta_{x}\right\}_{x \in V}$ the following components

$$
\Delta(x, y)= \begin{cases}\sigma_{x y} / \mu_{x}, & x \neq y \\ -1, & x=y\end{cases}
$$

Although this matrix is not symmetric, in another basis $\left\{\mu_{x}^{-1 / 2} \delta_{x}\right\}_{x \in V}$, the Laplace operator is represented by a symmetric matrix

$$
\widetilde{\Delta}(x, y)= \begin{cases}\sigma_{x y}\left(\mu_{x} \mu_{y}\right)^{-1 / 2}, & x \neq y \\ -1, & x=y\end{cases}
$$

See [10] for more details. 


\subsection{Green's formula.}

The following discrete analogue of the Green formula is true. If $\Omega$ is a finite subset of $V$ and $f$ is a function on $\Omega$ then

$$
\sum_{x \in \Omega} \Delta f(x) \mu_{x}=\sum_{\substack{x \in \Omega \\ y \notin \Omega}}\left(\nabla_{x y} f\right) \sigma_{x y}=\sum_{e \in \partial \Omega}\left(\nabla_{e} f\right) \sigma_{e} .
$$

If $f$ and $g$ are functions on $V$ and one of them has a finite support then

$$
\sum_{x \in V} \Delta f(x) g(x) \mu_{x}=-\frac{1}{2} \sum_{x, y \in V}\left(\nabla_{x y} f\right)\left(\nabla_{x y} g\right) \sigma_{x y}=-\sum_{e \in E}\left(\nabla_{e} f\right)\left(\nabla_{e} g\right) \sigma_{e} .
$$

The multiple $\frac{1}{2}$ appears in the middle term because each edge counts twice. In the third term, each edge may be oriented arbitrarily because the product $\left(\nabla_{e} f\right)\left(\nabla_{e} g\right)$ does not depend on the choice of orientation.

\subsection{Integration versus Summation.}

Most of the proofs in the case of graphs follow the same line of arguments as in the case of manifolds. This becomes especially clear if we write down the basic facts from analysis on graphs in terms of integration against the measures $\mu$ and $\sigma$. By suppressing the subscripts of $\mu, \sigma$ and $\nabla$, we can write

$$
\begin{aligned}
\sum_{x \in V} f(x) \mu_{x} & =\int_{V} f d \mu, \\
\sum_{e \in E}\left|\nabla_{e} f\right| \sigma_{e} & =\int_{E}|\nabla f| d \sigma, \\
\sum_{e \in E}\left(\nabla_{e} f\right)\left(\nabla_{e} g\right) \sigma_{e} & =\int_{E} \nabla f \nabla g d \sigma,
\end{aligned}
$$

where $f, g$ are functions on $V$.

The Green formula (5.3) can be rewritten, by using the integration, as follows

$$
\int_{V} \Delta f g d \mu=-\int_{E} \nabla f \nabla g d \sigma
$$

provided one of the functions $f, g$ has finite support. 


\subsection{Eigenvalues of Laplacian.}

Let $\Omega$ be a finite subset of vertices of a weighted graph $(\Gamma, \sigma)$. Denote by $C_{0}(\Omega)$ a class of functions on $V$ which vanish outside $\Omega$. We say that a function $f$ defined on $V$ satisfies the Dirichlet boundary condition in $\Omega$ if $f \in C_{0}(\Omega)$. Denote by $\Delta_{\Omega}$ restriction of the Laplace operator $\Delta$ to the space $C_{0}(\Omega)$ that is,

$$
\Delta_{\Omega} f(x)= \begin{cases}\Delta f(x), & x \in \Omega \\ 0, & x \notin \Omega .\end{cases}
$$

The operator $\Delta_{\Omega}$ is referred to as the Laplace operator with the Dirichlet boundary condition in $\Omega$.

As follows easily from the Green formula (5.4), for all $f, g \in C_{0}(\Omega)$,

$$
\int_{\Omega}\left(\Delta_{\Omega} f\right) g d \mu=-\int_{E} \nabla f \nabla g d \sigma=\int_{\Omega}\left(\Delta_{\Omega} g\right) f d \mu .
$$

In particular, the operator $\Delta_{\Omega}$ is symmetric with respect to the measure $\mu_{x}$ and negative definite.

Let $|\Omega|$ denote the number of vertices in $\Omega$. The space $C_{0}(\Omega)$ has the finite dimension $|\Omega|$. Therefore, the operator $-\Delta_{\Omega}$ has a real positive ${ }^{5}$ spectrum consisting of $|\Omega|$ eigenvalues $\lambda_{1} \leq \lambda_{2} \cdots \leq \lambda_{|\Omega|}$. The corresponding eigenfunctions $\phi_{i} \in C_{0}(\Omega)$ are called the Dirichlet eigenfunctions of $\Omega$.

The first eigenvalue $\lambda_{1}(\Omega)$ can also be defined by the variational principle

$$
\lambda_{1}(\Omega)=\inf _{\substack{f \in C_{0}(\Omega) \\ f \neq 0}} \frac{\int_{E}|\nabla f|^{2} d \sigma}{\int_{V} f^{2} d \mu} .
$$

\subsection{Heat kernel.}

Given a finite set $\Omega \subset V$, we introduce the heat kernel $p_{\Omega}(t, x, y)$ (where $x, y \in V$ and $t \geq 0$ ) as the kernel of the operator $e^{t \Delta_{\Omega}}$ with respect to the measure $\mu$. In other words, for any function $f \in C_{0}(\Omega)$,

$$
e^{t \Delta_{\Omega}} f(x)=\int_{V} p_{\Omega}(t, x, y) f(y) d \mu_{y} .
$$

The following are the basic properties of the heat kernel $p_{\Omega}$ which follow directly from the definition.

\footnotetext{
${ }^{5}$ Strictly speaking, this is true if $\Omega$ is a proper subset of $V$. If $\Omega=V$ then $|\Omega|<\infty$ implies $\lambda_{1}(\Omega)=0$ because the constant function is the first eigenfunction.
} 
1. $p_{\Omega}(t, x, y) \geq 0$; moreover, if $x$ or $y \notin \Omega$ then $p_{\Omega}(t, x, y)=0$.

2. $p_{\Omega}(t, x, y)=p_{\Omega}(t, y, x)$.

3. For a fixed vertex $y, p_{\Omega}(t, x, y)$ as a function of $x \in \Omega$ and $t \geq 0$, satisfies the heat equation

$$
\frac{\partial}{\partial t} p_{\Omega}(t, x, y)=\Delta p_{\Omega}(t, x, y)
$$

4. $p(0, x, \cdot)=\mu_{x}^{-1} \delta_{x}$.

5. If $\left\{\phi_{i}\right\}_{i=1}^{|\Omega|}$ are the eigenfunctions of $-\Delta_{\Omega}$ which form an orthonormal basis in $L^{2}(\Omega, \mu)$, and $\left\{\lambda_{i}\right\}$ are the corresponding eigenvalues then

$$
p_{\Omega}(t, x, y)=\sum_{i=1}^{|\Omega|} e^{-t \lambda_{i}} \varphi_{i}(x) \varphi_{i}(y) .
$$

In particular, it implies the trace formula

$$
\int_{V} p_{\Omega}(t, x, x) d \mu_{x}=\sum_{i=1}^{|\Omega|} e^{-t \lambda_{i}} .
$$

6. For all $x$ and $t \geq 0$,

$$
\int_{V} p_{\Omega}(t, x, y) d \mu_{y} \leq 1
$$

7. For all $x, y$ and $0 \leq s \leq t$,

$$
p_{\Omega}(t, x, y)=\int_{V} p_{\Omega}(s, x, z) p_{\Omega}(t-s, z, y) d \mu_{z} .
$$

\subsection{Co-area formula.}

The co-area formula (3.5) has a well-known discrete analogue (see, for example [5], [10], [29], [32]).

Proposition 5.1. Given any function $f$ on $V$, let us denote

$$
\Omega_{t}=\{x \in V: f(x)>t\} .
$$

Then

$$
\int_{E}|\nabla f| d \sigma=\int_{-\infty}^{\infty} \sigma\left(\partial \Omega_{t}\right) d t .
$$


Proof. For any edge $e:=x y \in E$, there corresponds an interval $I_{e} \subset \mathbb{R}$ which is defined as follows:

$$
I_{e}=[f(y), f(x)) \quad \text { if } f(x) \geq f(y) .
$$

Also, let us denote

$$
\sigma\left(I_{e}\right)=\sigma_{x y}
$$

Then we have

$$
\int_{E}|\nabla f| d \sigma=\frac{1}{2} \sum_{\{x, y: x \sim y\}}|f(x)-f(y)| \sigma_{x y}=\sum_{e \in E}\left|I_{e}\right| \sigma\left(I_{e}\right)
$$

where $\left|I_{e}\right|$ is the Euclidean length of the interval $I_{e}$.

The boundary $\partial \Omega_{t}$ consists of edges $e=x y$ such that $x \in \Omega_{t}$ and $y \notin \Omega_{t}$. This is equivalent to $f(x)>t$ and $f(y) \leq t$ which, in turn, can be written as $t \in I_{e}$. Thus, we have

$$
\sigma\left(\partial \Omega_{t}\right)=\sum_{e \in \partial \Omega_{t}} \sigma_{e}=\sum_{\left\{e: I_{e} \ni t\right\}} \sigma\left(I_{e}\right)=\sum_{e \in E} \sigma\left(I_{e}\right) \mathbf{1}_{I_{e}}(t) .
$$

Finally, by interchanging the summation and the integration, we obtain

$$
\int_{-\infty}^{+\infty} \sigma\left(\partial \Omega_{t}\right) d t=\sum_{e \in E} \int_{-\infty}^{+\infty} \sigma\left(I_{e}\right) \mathbf{1}_{I_{e}}(t) d t=\sum_{e \in E} \sigma\left(I_{e}\right)\left|I_{e}\right|
$$

whence (5.5) follows.

\section{Discrete isoperimetric inequality.}

\subsection{Statement of the results.}

The purpose of this section is to prove a graph analogue of Theorem 2.1. As was mentioned in Introduction, in other to do so we need some more structure on the graph.

We will consider distance functions on $V$. The simplest example of a distance function on $V$ is the graph distance which is, by definition, the number of edges in the shortest edge path joining two points of $V$. We assume that we are given some distance function on $V$ and denote it by $\rho_{\xi}(x)$, where $x, \xi \in V$. Denote by $B_{\xi}(x)$ the ball defined by $\rho$, that is,

$$
B_{\xi}(r)=\left\{x: \rho_{\xi}(x)<r\right\} .
$$




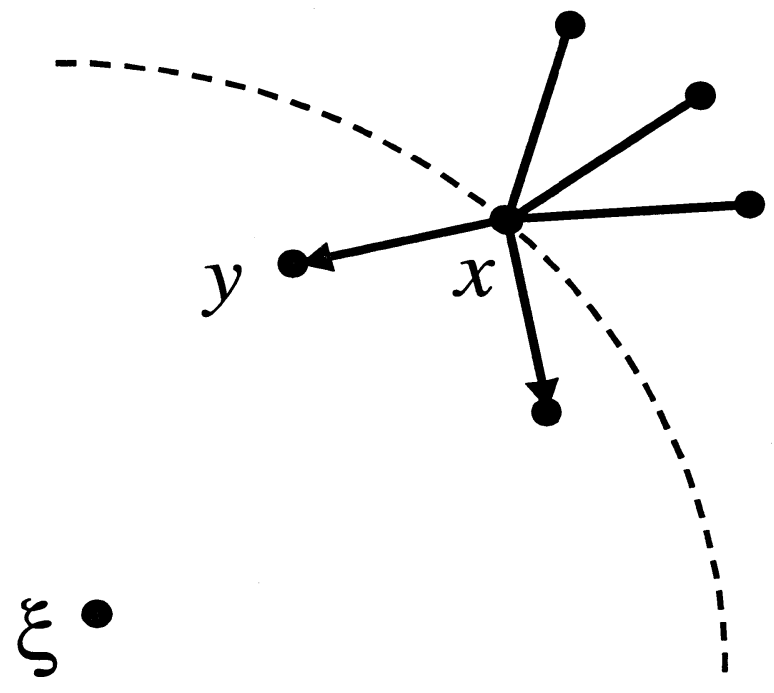

Figure 9: Example: $\mu_{x}^{(\xi)}=2$ and $\mu_{x}=5$

Let us assume that $\rho_{\xi}$ has the following property:

$$
\left|\nabla_{x y} \rho_{\xi}\right| \leq 1
$$

for any edge $x y \in E$ and for any vertex $\xi \in V$. For example, (6.1) obviously holds for the graph distance.

Next, we will need the following constant characterizing a structure of edges at the boundary of the ball $B_{\xi}(r)$. Given points $\xi, x \in V$, consider the following sum of $\sigma_{x y}$ over all points $y$ adjacent to $x$ and satisfying $\rho_{\xi}(y)<$ $\rho_{\xi}(x)$ :

$$
\mu_{x}^{(\xi)}=\sum_{\left\{y: y \sim x \text { and } \rho_{\xi}(y)<\rho_{\xi}(x)\right\}} \sigma_{x y}
$$

Clearly, $\mu_{x}^{(\xi)} \leq \mu_{x}$ (see Fig. 9). We define the spring ratio $\nu_{r}$, for any $r>0$, as follows

$$
\nu_{r}=\inf _{\substack{\xi \in M \\ x \in B_{\xi}(r)}} \frac{\mu_{x}}{\mu_{x}^{(\xi)}}
$$




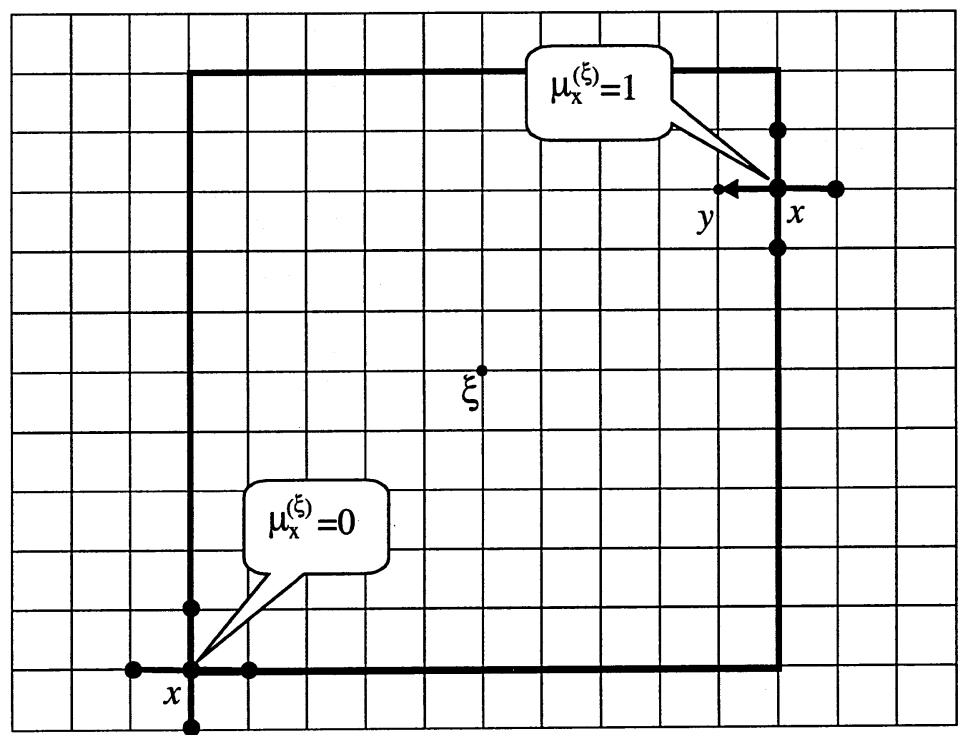

Figure 10: $\mathbb{Z}^{2}$ with the metric $\rho_{\xi}(x)=\max _{i}\left|x_{i}-\xi_{i}\right|$ has the spring ratio $\nu_{r}=4\left(\mu_{x}=4\right.$ and $\left.\max \mu_{x}^{(\xi)}=1\right)$

Example 6.1. We consider the graph $(\Gamma, \sigma)$ with the vertex set $\mathbb{Z}^{n}$ and with the measure

$$
\sigma_{x y}= \begin{cases}1, & \text { if } x \text { and } y \text { differ exactly at one coordinate by } 1, \\ 0, & \text { otherwise. }\end{cases}
$$

For vertices $x, \xi \in \mathbb{Z}^{n}$, we define the distance by

$$
\rho_{\xi}(x)=\max _{1 \leq i \leq n}\left|x_{i}-\xi_{i}\right| \text {. }
$$

Clearly, $\left|\nabla_{x y} \rho_{\xi}(x)\right| \leq 1$ and (6.1) is satisfied. The $\rho$-ball $B_{\xi}(r)$ is a cube centered at $\xi$ and with the sides parallel to the coordinate axis. Let us find the spring ratio $\nu_{r}$ defined by (6.3). Assume for simplicity that $\xi$ is the origin. Any point $x \neq \xi$ has at most one neighbor $y$ such that $\max _{i}\left|y_{i}\right|<\max _{i}\left|x_{i}\right|$, that is $\rho_{\xi}(y)<\rho_{\xi}(x)$. Thus, $\mu_{x}^{(\xi)}=1$ or 0 . Since $\mu_{x}=2 n$, we conclude $\nu_{r}=2 n$ (see Fig. 10).

Together with the function $\rho_{\xi}(x)$, we consider another function $q_{\xi}(x)$ an analogue of the square distance. We postulate the following properties of $q$, for some positive constants $\delta, \iota$ and $R_{0}$ :

(i) $q_{\xi}(x) \geq 0$, and $q_{\xi}(x)=0$ if and only if $x=\xi$. 
(ii) For any vertex $\xi$ and for arbitrary adjacent vertices $x, y \in B_{\xi}\left(R_{0}\right)$,

$$
\nabla_{x y} q_{\xi} \leq \rho_{\xi}(x)+\iota
$$

clearly, we can always assume that

$$
\iota \geq 1 \text {. }
$$

(iii) For any vertex $\xi$ and all $x \in B_{\xi}\left(R_{0}\right)$,

$$
\Delta q_{\xi}(x) \geq \delta \text {. }
$$

Example 6.2. Let $(\Gamma, \sigma)$ be the rectangular lattice graph defined on $\mathbb{Z}^{n}$ as in Example 6.1. Let us consider

$$
\rho_{\xi}(x)=\max _{1 \leq i \leq n}\left|x_{i}-\xi_{i}\right|
$$

and

$$
q_{\xi}(x)=\frac{1}{2} \sum_{i=1}^{n}\left(x_{i}-\xi_{i}\right)^{2} .
$$

In other word, $\rho_{\xi}(x)$ is the $l^{\infty}$-distance whereas $q_{\xi}(x)$ is determined by the $l^{2}$-distance. Condition (i) is obvious. Let us verify (ii) and (iii), assuming for simplicity that $\xi$ is the origin.

For $x \sim y$, we know that $y$ is obtained from $x$ by changing by 1 of one of the coordinates of $x$. Let this coordinate be $x_{1}$. Since $\left|x_{1}\right| \leq \rho_{\xi}(x)$, we obtain (see Fig. 11)

$$
\nabla_{x y} q_{\xi}=\frac{1}{2}\left(\left(x_{1} \pm 1\right)^{2}+x_{2}^{2}+\ldots+x_{n}^{2}\right)-\frac{1}{2} \sum_{i=1}^{n} x_{i}{ }^{2}= \pm x_{1}+\frac{1}{2} \leq \rho_{\xi}(x)+\frac{1}{2}
$$

whence (ii) follows.

To verify (iii), for any $y \sim x$, we denote by $y^{\prime}$ the vertex symmetric to $y$ with respect to $x$.

The contribution of the points $y$ and $y^{\prime}$ to the sum (5.1), defining the Laplace operator $\Delta q_{\xi}(x)$, is equal to (see Fig. 12)

$$
q_{\xi}(y)+q_{\xi}\left(y^{\prime}\right)-2 q_{\xi}(x)=\frac{1}{2}|y|^{2}+\frac{1}{2}\left|y^{\prime}\right|^{2}-\left|\frac{y+y^{\prime}}{2}\right|^{2}=\left|\frac{y-y^{\prime}}{2}\right|^{2}=1 .
$$




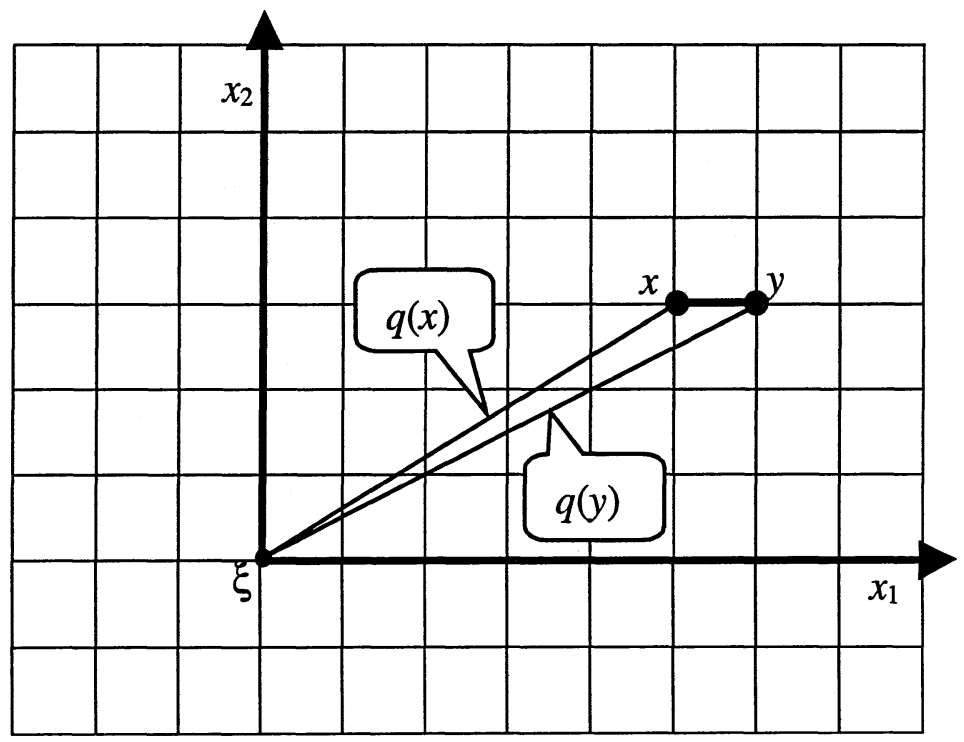

Figure 11: Computing $\nabla_{x y} q_{\xi}$

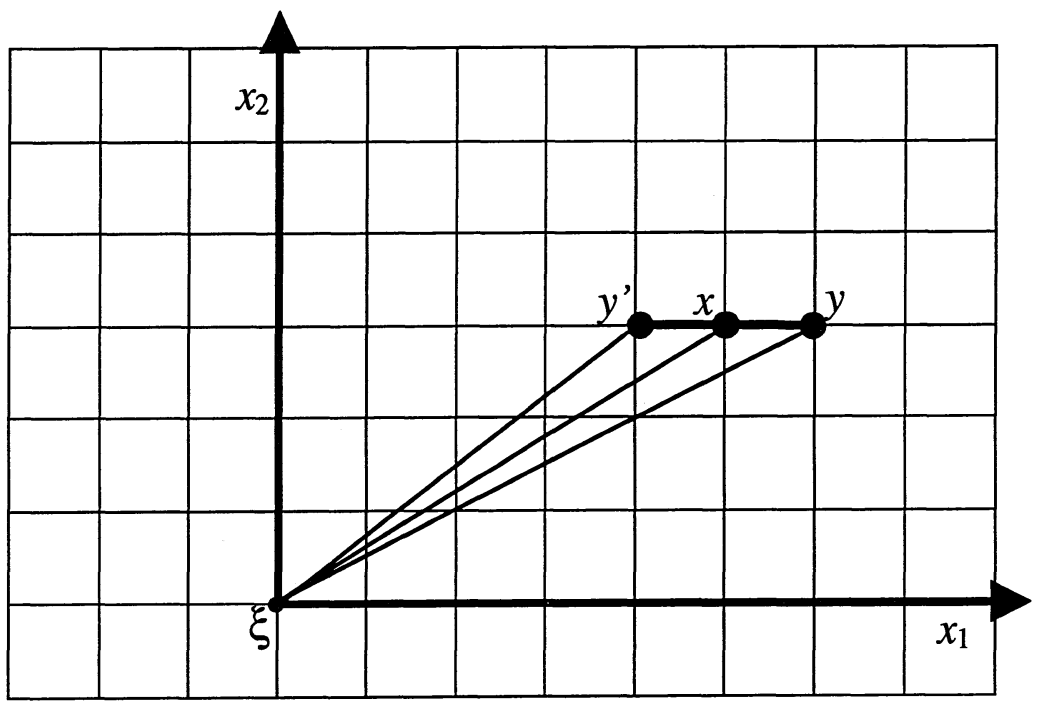

Figure 12: Computing $\Delta q_{\xi}(x)$ 
Since we have $n$ such pairs of neighbors of $x$, and $\mu_{x}=2 n$, we see that

$$
\Delta q_{\xi}(x)=\frac{1}{2}
$$

and (6.7) is satisfied with $\delta=1 / 2$.

We remark that neither of other natural choices of $q$ such as

$$
q(x)=\frac{1}{2}\left(\sum_{i}\left|x_{i}\right|\right)^{2}
$$

or

$$
q(x)=\frac{1}{2} \max \left|x_{i}\right|^{2},
$$

admits both (6.5) and (6.7) with $\delta \geq 1 / 2$.

Definition. Given positive numbers $\delta, \iota$ and $R_{0}$, we say that a weighted graph $(\Gamma, \sigma)$ has property $P\left(\delta, \iota, R_{0}\right)$ if there exist a distance function $\rho$ satisfying (6.1) and a function $q$ satisfying the hypotheses (i)-(iii) such that

$$
n:=\delta \nu_{R_{0}+1} \geq 1,
$$

where $\nu_{R_{0}+1}$ and $\delta$ come from (6.3) and (6.7) respectively.

The claim that a graph has property $P\left(\delta, \iota, R_{0}\right)$ contains two different issues. First, it means the existence of functions $\rho$ and $q$ as above. Second, it means that the number $n$ defined by (6.8) is greater than or equal to 1 . In particular, a small $\delta$ in (6.7) is allowed provided it is compensated by a large spring ratio $\nu_{R_{0}+1}$ defined by (6.3). In what follows, the number $n$ will play the role of the isoperimetric dimension.

It will be more convenient for us to replace the argument $\delta$ in $P\left(\delta, \iota, R_{0}\right)$ by $n$ because $\delta$ will be used only through $n$. So, by somewhat abusing the notation, we will refer to the property $P\left(n, \iota, R_{0}\right)$ rather than to $P\left(\delta, \iota, R_{0}\right)$. The value of the number $\iota$ is not of much importance. We will assume $\iota \geq 1$ as in (6.6).

Note that Proposition 6.5 in the next section states that the property $P\left(n, \iota, R_{0}\right)$ implies the following lower bound of the volume of any ball of radius $r<R_{0}$ :

$$
\mu\left(B_{\xi}(r)\right) \geq b \mu_{\xi} r^{n},
$$

where $b=b(\iota, n)>0$. Moreover, the following isoperimetric inequality is true. 
Theorem 6.3. Assume that the weighted graph $(\Gamma, \sigma)$ has property $P\left(n, \iota, R_{0}\right)$. Assume also that

$$
\omega^{\prime}:=\inf _{x \sim y} \sigma_{x y}>0
$$

and denote

$$
\omega:=\inf _{x \in V} \mu_{x} .
$$

Let $\Omega$ be a finite subset of $V$ such that

$$
\mu(\Omega) \leq \frac{\omega}{\theta} R_{0}^{n}
$$

where

$$
\theta:=8 \iota^{n} e^{2 n^{2}}
$$

Then

$$
\sigma(\partial \Omega) \geq c \mu(\Omega)^{1-1 / n}
$$

where $c:=4^{-n-3} \nu_{R_{0}+1}^{-1} \iota^{-1} e^{-2 n} \omega^{\prime} \omega^{1 / n-1}$.

As an immediate consequence of Theorem 6.3 (with $R_{0}=\infty$ ) and Examples 6.1 and 6.2 , we have the following:

Corollary 6.4. For the lattice graph on $\mathbb{Z}^{n}$, for any finite subset $\Omega$ of vertices, we have

$$
\sigma(\partial \Omega) \geq c \mu(\Omega)^{1-1 / n}
$$

where $c=c(n)>0$.

The constant $c$ obtained in this way is not optimal. See [30] for the sharp constant $c$ in $\mathbb{Z}^{n}$.

Let us emphasize that Theorem 6.3 is not a straight analogue of Theorem 2.1. To start with, in the setting of manifolds, we needed only one distance function $\rho$. However, also for manifolds, we can choose the function $q$ by

$$
q=\frac{1}{2} \rho^{2} .
$$




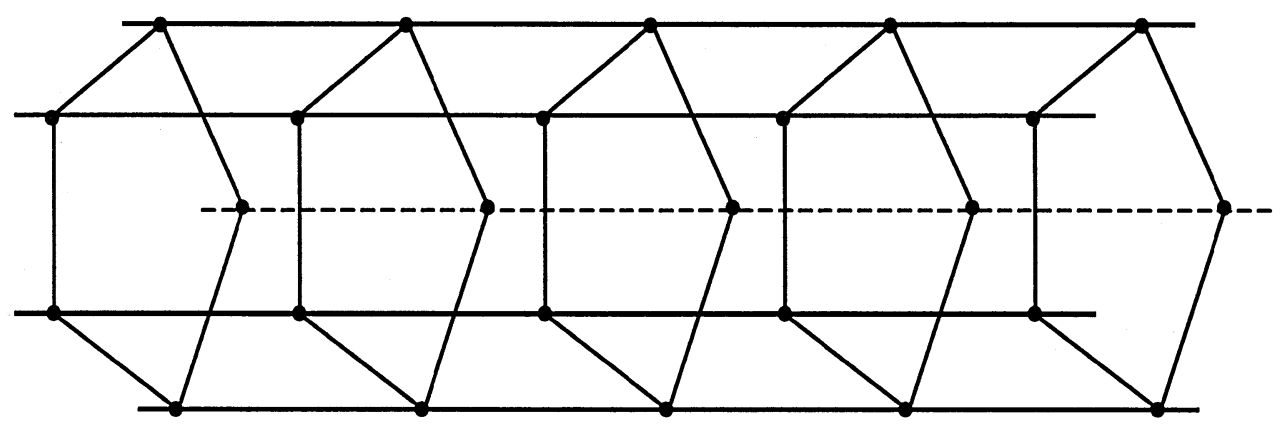

Figure 13: Graph $C_{5} \times \mathbb{Z}^{1}$

We have then $|\nabla q| \leq \rho$ which follows from $|\nabla \rho| \leq 1$ and which is the analogue of (6.5). The hypothesis (2.2) takes the form

$$
\Delta q \geq n
$$

which is the analogue of (6.7). The reason why we do not assume the relation (6.13) for the graphs, is that we do not know whether there exists a function $\rho$ on $\mathbb{Z}^{n}$ such that (6.5) and (6.7) hold with $q=\frac{1}{2} \rho^{2}$.

There is a more substantial difference between the continuous and discrete case. In the case of manifolds, the isoperimetric dimension $n$ comes directly from the lower bound of $\Delta q$ as in (6.14). In the case of graphs, $n$ is the product of two quantities $\delta$ and $\nu=\nu_{R_{0}+1}$ that come from different hypotheses. The spring ratio $\nu$ has no analogue for manifolds. It is clear that the Laplace operator of the distance function on a graph does not catch the isoperimetric dimension as we have seen for $\mathbb{Z}^{n}$. The spring ratio $\nu$ helps "to see" the dimension.

One may wonder if it is possible to catch the sharp isoperimetric inequality only by using the spring ratio. The answer is negative.

Indeed, let us consider graph $\Gamma$ which is the direct product of a cycle $C_{k}$ with an odd $k$ and $\mathbb{Z}^{1}$ (see Fig. 13); in other words, $\Gamma$ is a discrete cylinder based on $C_{k}$. Let the distance $\rho$ on $\Gamma$ be the maximum of the graph distances in $C_{k}$ and in $\mathbb{Z}^{1}$, analogously to (6.4). It is easy to see that $\nu_{r}=4$ for this graph (for any $r>1$ ) exactly as for $\mathbb{Z}^{2}$. However, the isoperimetric dimensions of $\mathbb{Z}^{2}$ and $C_{k} \times \mathbb{Z}^{1}$ are clearly different.

The rest of this Section is devoted to the proof of Theorem 6.3. 


\subsection{Boundary of a ball.}

We start with the following statement establishing relation between the boundary area of a ball and its volume.

Proposition 6.5. Assume that the weighted graph $(\Gamma, \sigma)$ has property $P\left(n, \iota, R_{0}\right)$. Then, for any value $r \in\left[0, R_{0}\right)$ and any vertex $\xi \in V$, we have

$$
\sigma\left(\partial B_{\xi}(r)\right) \geq \frac{\delta}{r+\iota} \mu\left(B_{\xi}(r)\right)
$$

and

$$
\mu\left(B_{\xi}(r)\right) \geq b \mu_{\xi} r^{n}
$$

where

$$
b=\iota^{-n} \exp \left(-n^{2}\right)
$$

Proof. For simplicity, we will write $\nu=\nu_{R_{0}+1}$ and $B(r)=B_{\xi}(r)$. To prove (6.15), we sum up the inequality (6.7) pre-multiplied by $\mu_{x}$, over the ball $B(r)$ and use the Green formula (5.2). We obtain

$$
\delta \mu(B(r)) \leq \sum_{x \in B(r)} \Delta q(x) \mu_{x}=\sum_{\substack{x \in B(r) \\ y \notin B(r)}}\left(\nabla_{x y} q\right) \sigma_{x y} .
$$

Of course, the summation on the right-hand side of (6.18) is restricted to adjacent $x, y$. Therefore, we can use the hypothesis (6.5), which states that

$$
\nabla_{x y} q \leq \rho(x)+\iota \leq r+\iota .
$$

Thus, (6.18) implies

$$
\delta \mu(B(r)) \leq(r+\iota) \sigma(\partial B(r))
$$

which proves (6.15).

Next we prove that, for all $r \in\left[0, R_{0}\right)$,

$$
\mu(B(r+1))-\mu(B(r)) \geq \frac{n}{r+\iota} \mu(B(r)) .
$$




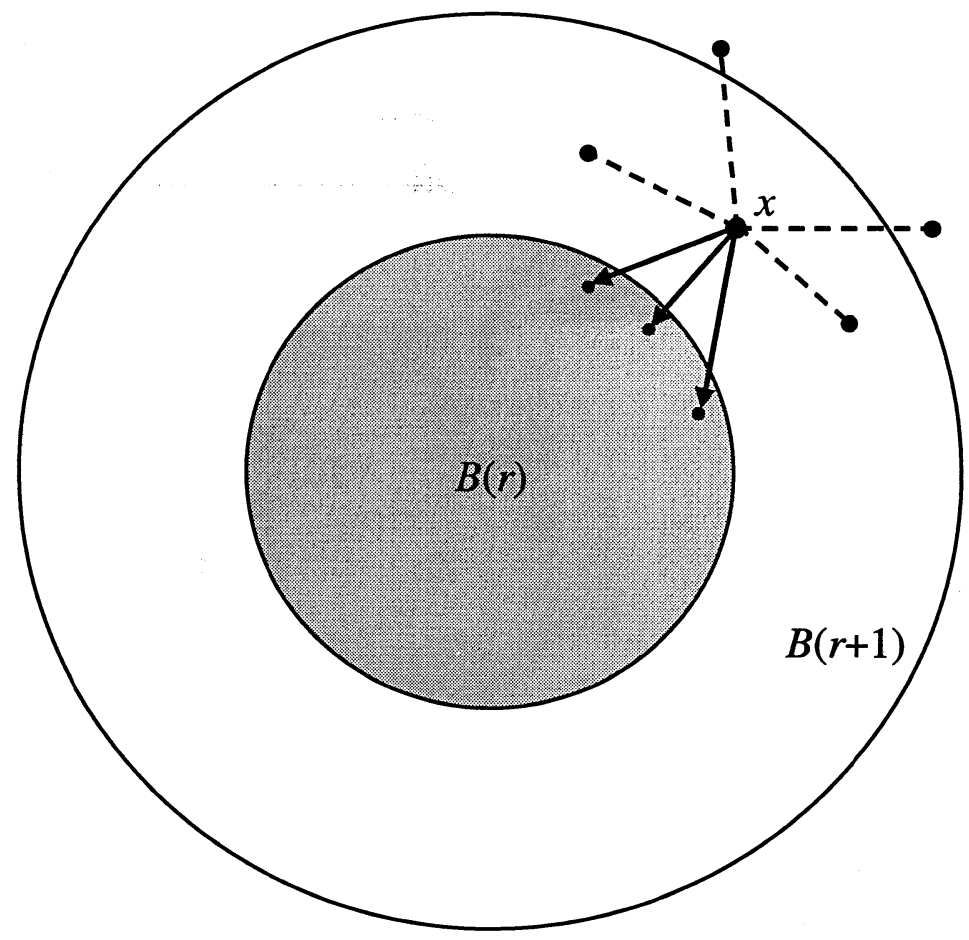

Figure 14: The edges at $x$ contributing to $\sigma(\partial B(r))$ are bold whereas the others are dashed

In the view of (6.19) and (6.8), inequality (6.20) will follow from

$$
\sigma(\partial B(r)) \leq \frac{1}{\nu}(\mu(B(r+1))-\mu(B(r)))
$$

where we write $\nu=\nu_{R_{0}+1}$.

Let us verify (6.21). The hypothesis (6.1) implies that if $x$ is adjacent to $y \in B(r)$ then $x \in B(r+1)$. Therefore, any edge $e \in \partial B(r)$ connects a vertex $y \in B(r)$ with a vertex $x \in B(r+1) \backslash B(r)$ whence

$$
\sigma(\partial B(r)) \leq \sum_{\substack{y \in B(r) \\ x \in B(r+1) \backslash B(r)}} \sigma_{x y} .
$$

Fix a vertex $x \in B(r+1) \backslash B(r)$ and consider all edges $e$ which join $x$ with a vertex $y$ in $B(r)$ (see Fig. 14). 
The sum of $\sigma_{x y}$ over all such $y$ is at most $\mu_{x}^{(\xi)}$ as defined by (6.2). Therefore, we have

$$
\sigma(\partial B(r)) \leq \sum_{x \in B(r+1) \backslash B(r)} \mu_{x}^{(\xi)}
$$

From the definition (6.3) of the spring ratio, we know that

$$
\mu_{x}^{(\xi)} \leq \frac{\mu_{x}}{\nu}
$$

Hence,

$$
\sigma(\partial B(r)) \leq \frac{1}{\nu} \sum_{x \in B(r+1) \backslash B(r)} \mu_{x}=\frac{1}{\nu} \mu(B(r+1) \backslash B(r)),
$$

which proves (6.21) and, thus, (6.20).

Clearly, (6.20) can be rewritten as

$$
\mu(B(r+1)) \geq\left(1+\frac{n}{r+\iota}\right) \mu(B(r)) .
$$

For an integer $r \in\left[1, R_{0}\right)$, we iterate (6.22) to obtain

$$
\mu(B(r)) \geq \mu(B(0)) \prod_{k=0}^{r-1}\left(1+\frac{n}{k+\iota}\right) .
$$

By using the elementary inequality $1+x \geq \exp \left(x-\frac{1}{2} x^{2}\right)(x>0), \mu(B(0))=$ $\mu_{\xi}$ and $\iota \geq 1$ (see (6.6)) we derive from (6.23)

$$
\begin{aligned}
\mu(B(r)) & \geq \mu_{\xi} \exp \left(n \sum_{k=0}^{r-1} \frac{1}{k+\iota}-\frac{n^{2}}{2} \sum_{k=0}^{r-1} \frac{1}{(k+\iota)^{2}}\right) \\
& \geq \mu_{\xi} \exp \left(n \int_{\iota}^{r+\iota} \frac{d s}{s}-\frac{n^{2}}{2} \sum_{k=0}^{\infty} \frac{1}{(k+1)^{2}}\right) \\
& \geq \mu_{\xi}\left(\frac{r+\iota}{\iota}\right)^{n} \exp \left(-n^{2}\right) \\
& \geq \mu_{\xi}\left(\frac{r+1}{\iota}\right)^{n} \exp \left(-n^{2}\right) .
\end{aligned}
$$

Observe that (6.24) is true also for $r=0$ because $\mu(B(0))=\mu_{\xi}$.

Finally, for any $r \in\left[0, R_{0}\right)$, we obtain

$$
\mu(B(r)) \geq \mu(B(\lfloor r\rfloor)) \geq \mu_{\xi}\left(\frac{r}{\iota}\right)^{n} \exp \left(-n^{2}\right)
$$

whence (6.16) follows. 


\subsection{Proof of isoperimetric inequality.}

Here we prove Theorem 6.3. Let us denote

$$
r_{0}=\left(\theta \frac{\mu(\Omega)}{\omega}\right)^{1 / n}
$$

where $\theta$ is defined by (6.11). Hypothesis (6.10) implies that $r_{0} \leq R_{0}$.

Let $\xi$ be any point in $\Omega$ (we may assume that $\Omega$ is not empty). By Proposition 6.5 and by the obvious inequality $b>\theta^{-1}$ (see (6.17) and (6.11)) we have

$$
\mu\left(B_{\xi}\left(R_{0}\right)\right) \geq b \mu_{\xi} R_{0}^{n}>\theta^{-1} \omega r_{0}^{n}=\mu(\Omega) .
$$

Therefore, the complement to $\Omega$ is not empty which implies that $\partial \Omega$ is not empty, too and, by (6.9), $\mu(\partial \Omega) \geq \omega^{\prime}$.

We write then

$$
\frac{\sigma(\partial \Omega)}{\mu(\Omega)^{\frac{n-1}{n}}} \geq \frac{\omega^{\prime}}{\left(\theta^{-1} \omega\right)^{\frac{n-1}{n}} r_{0}^{n-1}} .
$$

If we assume that $r_{0}$ is small enough, say, as follows

$$
r_{0} \leq 2 n^{2}+1
$$

then we obtain

$$
\sigma(\partial \Omega) \geq \theta^{\frac{n-1}{n}}\left(2 n^{2}+1\right)^{1-n} \omega^{\prime} \omega^{-\frac{n-1}{n}} \mu(\Omega)^{\frac{n-1}{n}} .
$$

It follows easily from (6.11) that

$$
\theta^{\frac{n-1}{n}}\left(2 n^{2}+1\right)^{1-n} \geq 1
$$

whence we conclude

$$
\sigma(\partial \Omega) \geq \omega^{\prime} \omega^{-\frac{n-1}{n}} \mu(\Omega)^{\frac{n-1}{n}},
$$

which proves (6.12) for $r_{0} \leq 2 n^{2}+1$.

We may assume in the sequel

$$
r_{0}>2 n^{2}+1 .
$$

The further proof will be split into three steps. 


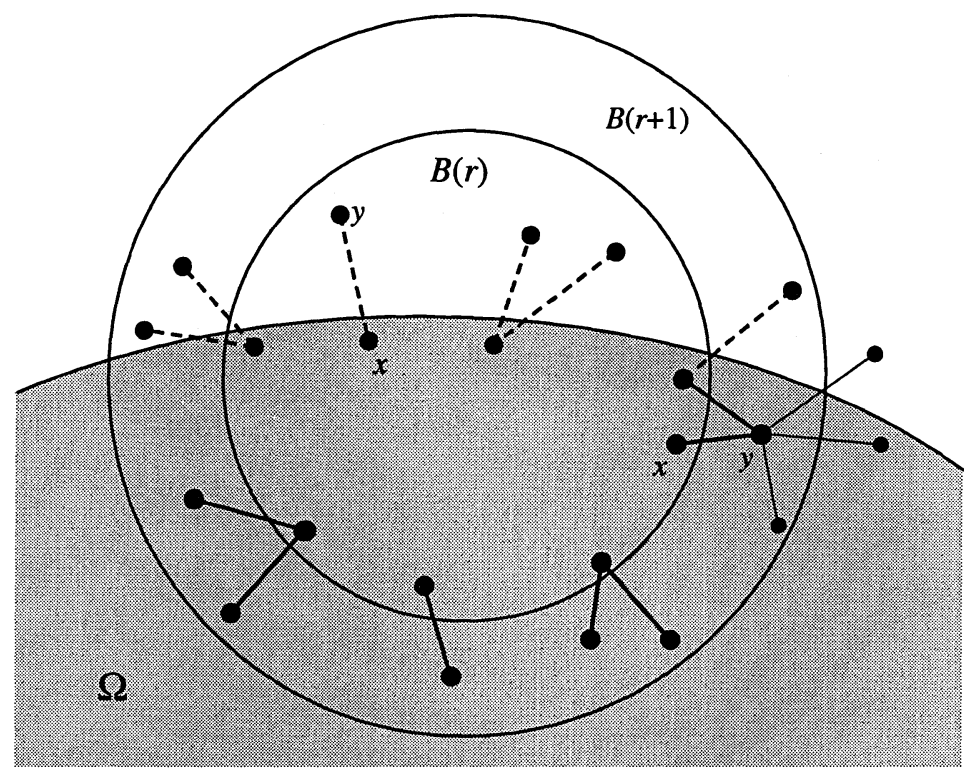

Figure 15: Splitting of $\partial(\Omega \cap B(r))$ - the dashed edges belong to $\partial^{+}(r)$, bold -to $\partial^{-}(r)$

STEP 1. The boundary $\partial\left(\Omega \cap B_{\xi}(r)\right)$ can be partitioned into two parts:

$$
\partial_{\xi}^{+}(r)=\left\{x y \in E: x \in \Omega \cap B_{\xi}(r) \text { and } y \notin \Omega\right\}
$$

and

$$
\partial_{\xi}^{-}(r)=\left\{x y \in E: x \in \Omega \cap B_{\xi}(r) \quad \text { and } \quad y \in \Omega \backslash B_{\xi}(r)\right\}
$$

(see Fig. 15). Denote

$$
m_{\xi}(r):=\mu\left(B_{\xi}(r) \cap \Omega\right) .
$$

We claim that the following inequality holds, for all $r \in\left[0, R_{0}\right]$,

$$
m_{\xi}(r)-m_{\xi}(r+1)+\frac{n}{r+\iota} m_{\xi}(r) \leq \nu \sigma\left(\partial_{\xi}^{+}(r)\right),
$$

where $\nu=\nu_{R_{0}+1}$.

The point $\xi$ will be fixed during the proof of (6.27) so we skip the subscript $\xi$ from all notation. As in the proof of Proposition 6.5, we integrate (6.7) over $\Omega \cap B(r)$ and obtain, by the Green formula (5.2),

$$
\delta m(r) \leq \sum_{x \in \Omega \cap B(r)} \Delta q(x) \mu_{x}=\sum_{x y \in \partial(\Omega \cap B(r))}\left(\nabla_{x y} q\right) \sigma_{x y} .
$$


For any edge $x y \in \partial(\Omega \cap B(r))$, we estimate $\nabla_{x y} q$ by using (6.5). Indeed, for $x \in \Omega \cap B(r)$, we have, by (6.5),

$$
\nabla_{x y} q_{\xi} \leq \rho_{\xi}(x)+\iota \leq r+\iota .
$$

Therefore, we obtain from (6.28)

$$
\delta m(r) \leq(r+\iota) \sigma(\partial(\Omega \cap B(r)))
$$

We now prove the following estimate of the measure of $\partial^{-}(r)$ :

$$
\sigma\left(\partial^{-}(r)\right) \leq \frac{1}{\nu}(m(r+1)-m(r)) .
$$

Indeed, for any edge $x y \in \partial^{-}(r)$ with $x \in B(r)$, (6.1) implies $y \in B(r+1)$. In order to add up $\sigma_{x y}$ over all $x y \in \partial^{-}(r)$, we first group those edges $x y$ with the same end $y$. Since $x \in B(r)$ and $y \notin B(r)$, the sum of $\sigma_{x y}$ over all possible $x$ (such that $x y \in \partial^{-}(r)$ ) is at most $\mu_{y}^{(\xi)}$, by (6.2). Thus,

$$
\begin{aligned}
\sigma\left(\partial^{-}(r)\right) & \leq \sum_{y \in \Omega \cap(B(r+1) \backslash B(r))} \mu_{y}^{(\xi)} \\
& \leq \frac{1}{\nu} \sum_{y \in \Omega \cap(B(r+1) \backslash B(r))} \mu_{y} \\
& =\frac{1}{\nu}(m(r+1)-m(r))
\end{aligned}
$$

where we have used $\mu_{y}^{(\xi)} \leq \frac{1}{\nu} \mu_{y}$ (see (6.3)).

By combining (6.29) and (6.30), we conclude

$$
\delta m(r) \leq(r+\iota) \sigma\left(\partial^{+}(r)\right)+\frac{r+\iota}{\nu}(m(r+1)-m(r)),
$$

whence (6.27) follows.

STEP 2. We claim that there exists $r \in\left[0, r_{0}\right]$ such that

$$
\sigma\left(\partial^{+}(r)\right) \geq \frac{\varepsilon}{r_{0}} m(4 r)
$$

where

$$
\varepsilon=4^{-n-1} \nu^{-1}
$$


Assume on the contrary that, for all $r \in\left[0, r_{0}\right]$, we have

$$
\sigma\left(\partial^{+}(r)\right)<\frac{\varepsilon}{r_{0}} m(4 r) .
$$

Together with (6.27), this yields

$$
m(r)-m(r+1)+\frac{n}{r+\iota} m(r) \leq \frac{\nu \varepsilon}{r_{0}} m(4 r) .
$$

Let us introduce a new function $\phi(r)=m(r-\iota) / r^{n}$, which is defined for all $r \geq \iota$. Replace $r$ by $r-\iota$ in (6.33) and rewrite the latter as follows:

$$
r^{n} \phi(r)-(r+1)^{n} \phi(r+1)+\frac{n}{r} r^{n} \phi(r) \leq \frac{\nu \varepsilon}{r_{0}}(4 r)^{n} \phi(4 r) .
$$

By dividing (6.34) by $(r+1)^{n}$ and by using (6.32), we obtain

$$
\left(\frac{r}{r+1}\right)^{n} \phi(r)-\phi(r+1) \leq \frac{1}{4 r_{0}}\left(\frac{r}{r+1}\right)^{n} \phi(4 r)-\frac{n}{r}\left(\frac{r}{r+1}\right)^{n} \phi(r) .
$$

Since, for any $x \in[0,1]$ and $n \geq 1$,

$$
1-x^{n} \leq n(1-x),
$$

we have

$$
1-\left(\frac{r}{r+1}\right)^{n} \leq \frac{n}{r+1}
$$

Therefore,

$$
\begin{aligned}
\phi(r)-\phi(r+1) & =\left(\frac{r}{r+1}\right)^{n} \phi(r)-\phi(r+1)+\left[1-\left(\frac{r}{r+1}\right)^{n}\right] \phi(r) \\
& \leq\left[\left(\frac{r}{r+1}\right)^{n} \phi(r)-\phi(r+1)\right]+\frac{n}{r+1} \phi(r),
\end{aligned}
$$

and (6.35) implies, for $\iota \leq r \leq \iota+r_{0}$,

$$
\begin{aligned}
\phi(r)-\phi(r+1) & \leq\left[\frac{1}{4 r_{0}}\left(\frac{r}{r+1}\right)^{n} \phi(4 r)-\frac{n}{r}\left(\frac{r}{r+1}\right)^{n} \phi(r)\right]+\frac{n}{r+1} \phi(r) \\
& \leq \frac{1}{4 r_{0}} \phi(4 r)+\frac{n}{r+1}\left[1-\left(\frac{r}{r+1}\right)^{n-1}\right] \phi(r) \\
& \leq \frac{1}{4 r_{0}} \phi(4 r)+\frac{n(n-1)}{(r+1)^{2}} \phi(r) .
\end{aligned}
$$


Thus, we have

$$
\phi(r)-\phi(r+1) \leq \frac{1}{4 r_{0}} \phi(4 r)+\frac{n^{2}}{(r+1)^{2}} \phi(r), \quad \forall r \in\left[\iota, r_{0}+\iota\right] .
$$

On the other hand, we know also that, for all $r \geq \iota$,

$$
\phi(r)=\frac{m(r-\iota)}{r^{n}} \leq \frac{\mu(\Omega)}{r^{n}}
$$

and

$$
\phi(r) \geq \frac{\mu(B(0))}{r^{n}} \geq \frac{\omega}{r^{n}} .
$$

We would like to bring to a contradiction using (6.36), (6.37) and (6.38). Denote

$$
\begin{aligned}
& L=\left\lceil 2 n^{2}+\iota\right\rceil, \\
& R=\left\lfloor r_{0}+\iota\right\rfloor .
\end{aligned}
$$

By (6.26), (6.39) and (6.40), we have

$$
\iota<L<R \leq r_{0}+\iota .
$$

Let us set

$$
M:=\max _{\substack{r \in[L, R] \\ r \text { is integer }}} \phi(r) .
$$

By iterating (6.36), we obtain, for any integer $r \in[L, R]$,

$$
\begin{aligned}
\phi(r)-\phi(R+1) & \leq \frac{1}{4 r_{0}} \sum_{k=r}^{R} \phi(4 k)+n^{2} \sum_{k=r}^{R} \frac{\phi(k)}{(k+1)^{2}} \\
& \leq \frac{1}{4 r_{0}} \sum_{k=L}^{4 R} \phi(k)+n^{2} \sum_{k=L}^{R} \frac{M}{(k+1)^{2}} \\
& \leq \frac{1}{4 r_{0}}\left[\sum_{k=R+1}^{4 R}+\sum_{k=L}^{R}\right] \phi(k)+n^{2} \frac{M}{L} \\
& \leq \frac{3 R}{4 r_{0}} \frac{\mu(\Omega)}{(R+1)^{n}}+\frac{R-L+1}{4 r_{0}} M+n^{2} \frac{M}{L}
\end{aligned}
$$

where we have used (6.37) and (6.41). 
It follows from (6.40), (6.39) that $R+1 \geq r_{0}, R-L+1 \leq r_{0}$ and $L \geq 2 n^{2}$. Therefore, (6.42) implies

$$
\phi(r)-\phi(R+1) \leq \frac{3}{4} \frac{\mu(\Omega)}{r_{0}^{n}}+\left(\frac{1}{4}+\frac{1}{2}\right) M .
$$

Choose $r \in[L, R]$ so that $\phi(r)=M$ and apply (6.37) in the form

$$
\phi(R+1) \leq \frac{\mu(\Omega)}{(R+1)^{n}} \leq \frac{\mu(\Omega)}{r_{0}^{n}} .
$$

Hence, we obtain from (6.43)

$$
M \leq \frac{7}{4} \frac{\mu(\Omega)}{r_{0}^{n}}+\frac{3}{4} M,
$$

whence, by (6.25),

$$
M \leq 7 \frac{\mu(\Omega)}{r_{0}^{n}}=7 \frac{\omega}{\theta} .
$$

In particular, this implies

$$
\phi(L) \leq 7 \frac{\omega}{\theta}
$$

whereas, by (6.38),

$$
\phi(L) \geq \frac{\omega}{L^{n}} .
$$

On the other hand, definitions (6.11) of $\theta$ and (6.39) of $L$ imply

$$
\frac{\theta}{7}>\iota^{n} e^{2 n^{2}}>\iota^{n}\left(1+2 n+2 n^{2}\right)^{n} \geq\left(\iota+1+2 n^{2}\right)^{n}>L^{n}
$$

and we see that (6.44) and (6.45) contradict each other.

STEP 3. For each vertex $\xi \in \Omega$, we denote by $r_{\xi}$ the value of $r$ determined by (6.31). Therefore, by (6.31), we have

$$
\sigma\left(\partial_{\xi}^{+}(r)\right) \geq \frac{\varepsilon}{r_{0}} \mu\left(B_{\xi}\left(4 r_{\xi}\right) \cap \Omega\right) \quad \forall \xi \in \Omega,
$$

where

$$
\partial_{\xi}^{+}(r)=\left\{u v \in E: u \in \Omega \cap B_{\xi}(r) \text { and } v \notin \Omega\right\} .
$$

By Lemma 2.6, there exists a subset $T \subset \Omega$ such that 
(i) all balls $B_{x}\left(r_{x}\right)$, for $x \in T$, are disjoint;

(ii) the union of the balls $B_{x}\left(4 r_{x}\right)$, for $x \in T$, covers $\Omega$.

We claim that, for distinct $x, y \in T$, the sets of edges $\partial_{x}^{+}\left(r_{x}\right)$ and $\partial_{y}^{+}\left(r_{y}\right)$ are disjoint. This is not altogether trivial because the boundaries $\partial B_{x}\left(r_{x}\right)$ and $\partial B_{y}\left(r_{y}\right)$ may have a common edge (the filled edge on Fig. 16). However, if there is an edge $e \in \partial_{x}^{+}\left(r_{x}\right) \cap \partial_{y}^{+}\left(r_{y}\right)$ then the edge $e$ must have a vertex in $\Omega \cap B_{x}\left(r_{x}\right)$ and a vertex in $\Omega \cap B_{y}\left(r_{y}\right)$. Due to (i), these vertices are different. Therefore, both vertices of $e$ belong to $\Omega$ which contradicts the definition (6.47) of $\partial_{x}^{+}(r)$.

Hence, all sets $\partial_{x}^{+}\left(r_{x}\right), x \in T$, are disjoint whence

$$
\sum_{x \in T} \sigma\left(\partial_{x}^{+}\left(r_{x}\right)\right) \leq \sigma(\partial \Omega) .
$$

On the other hand, (ii) implies

$$
\mu(\Omega) \leq \sum_{x \in T} \mu\left(B_{x}\left(4 r_{x}\right) \cap \Omega\right) .
$$

Using (6.49), (6.46), (6.48), (6.25), we obtain

$$
\begin{aligned}
\mu(\Omega) & \leq \sum_{x \in T} \mu\left(B_{x}\left(4 r_{x}\right) \cap \Omega\right) \\
& \leq \frac{r_{0}}{\varepsilon} \sum_{x \in T} \sigma\left(\partial_{x}^{+}\left(r_{x}\right)\right) \\
& \leq \frac{r_{0}}{\varepsilon} \sigma(\partial \Omega) \\
& =\varepsilon^{-1} \mu(\Omega)^{1 / n}\left(\frac{\theta}{\omega}\right)^{1 / n} \sigma(\partial \Omega) .
\end{aligned}
$$

This implies together with (6.11), (6.32) and $\omega \geq \omega^{\prime}$ that

$$
\sigma(\partial \Omega) \geq \varepsilon \theta^{-1 / n} \omega^{1 / n} \mu(\Omega)^{1-1 / n} \geq 4^{-n-3} \nu^{-1} \iota^{-1} e^{-2 n} \omega^{\prime} \omega^{1 / n-1} \mu(\Omega)^{1-1 / n},
$$

which was to be proved.

\section{Eigenvalues and Sobolev inequality on graphs.}

Most proofs below follow the same lines as their continuous counterparts in the previous sections. We emphasize only those places of the proofs which require additional argument specific to the discrete setup. 


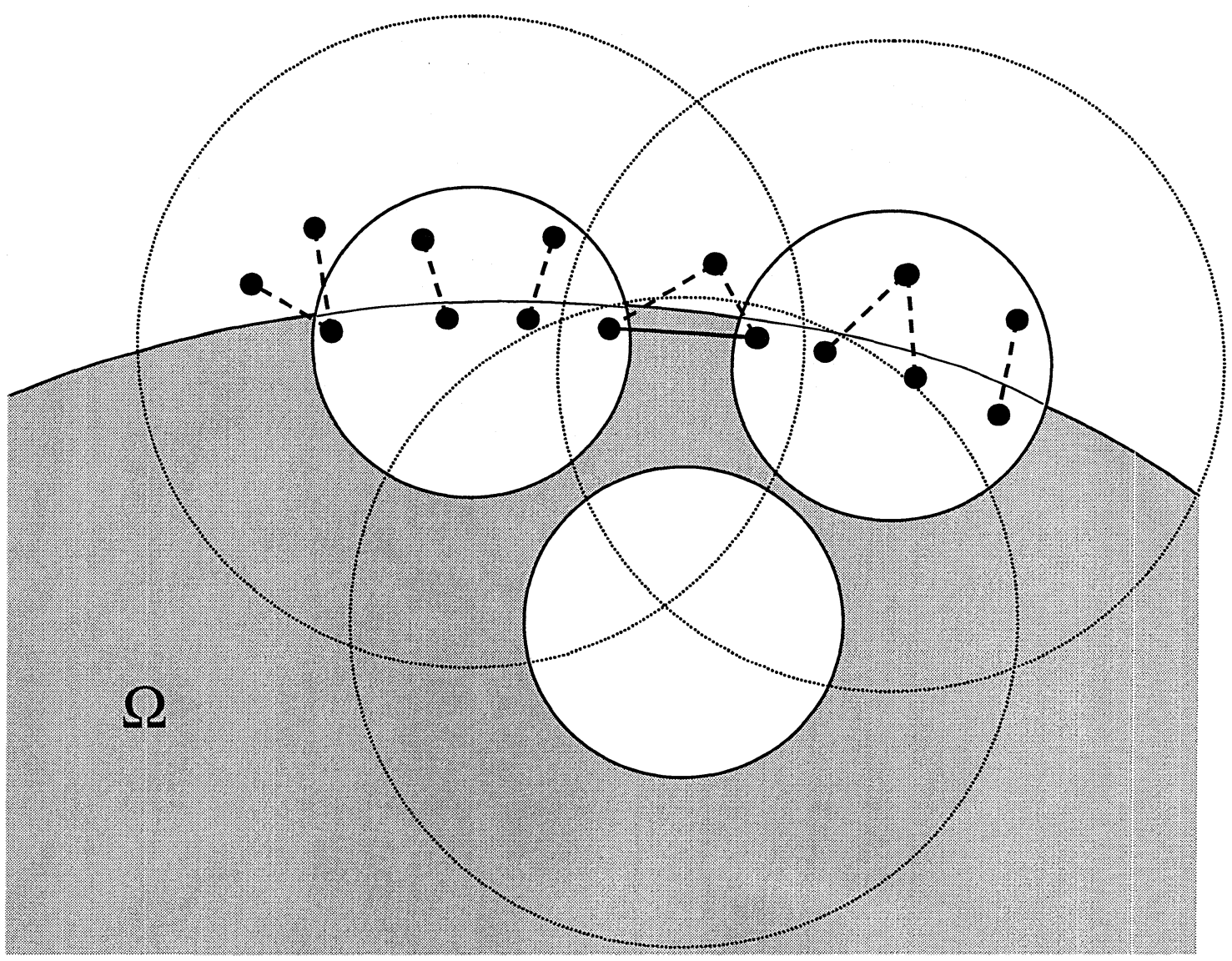

Figure 16: The boundaries $\partial_{x}^{+}\left(r_{x}\right), x \in T$, are disjoint (the dashed edges on the picture) whereas the balls $B_{x}\left(4 r_{x}\right)$ cover $\Omega$. 
Proposition 7.1. In a weighted graph $(\Gamma, \sigma)$, we assume that

$$
\sigma(\partial \Omega) \geq c \mu(\Omega)^{1-1 / n}
$$

for any $\Omega$ satisfying $\mu(\Omega) \leq v_{0}$, where $c>0, v_{0}>0$ and $n>1$. Then the Dirichlet eigenvalue $\lambda_{1}(\Omega)$ satisfies

$$
\lambda_{1}(\Omega) \geq \frac{c^{2}}{2} \mu(\Omega)^{-2 / n} .
$$

Proof. The proof is almost identical to that in Step 1 of Theorem 3.1. Only inequality (3.7), which is a part of Cheeger's argument, requires modification because we cannot use in the graph case the formula $\nabla f^{2}=2 f \nabla f$. However, the recipe is well known how to overcome this difficulty (see, for example, $[10],[12])$. So, we argue as follows:

$$
\begin{aligned}
\int_{E}\left|\nabla f^{2}\right| d \sigma & =\frac{1}{2} \sum_{x, y}\left|f^{2}(x)-f^{2}(y)\right| \sigma_{x y} \\
& =\frac{1}{2} \sum_{x, y}|f(x)-f(y)| \cdot|f(x)+f(y)| \sigma_{x y} \\
& \leq\left(\frac{1}{2}\left(\sum_{x, y}(f(x)-f(y))^{2} \sigma_{x y}\right) \cdot \frac{1}{2}\left(\sum_{x, y}(f(x)+f(y))^{2} \sigma_{x y}\right)\right)^{1 / 2} \\
& \leq\left(\left(\int_{E}|\nabla f|^{2} d \sigma\right) \cdot\left(\sum_{x} f^{2}(x) \mu_{x}+\sum_{y} f^{2}(y) \mu_{y}\right)\right)^{1 / 2} \\
& \leq\left(2\left(\int_{E}|\nabla f|^{2} d \sigma\right) \cdot\left(\int_{V} f^{2} d \mu\right)\right)^{1 / 2}
\end{aligned}
$$

This allows us to follow the rest of the proof as in the continuous case with a modified constant (off by a factor 2 ).

Next Lemma is a straight analogue of Lemma 3.2.

Lemma 7.2. In a weighted graph $(\Gamma, \mu)$, we assume that for any $\Omega$ satisfying $\mu(\Omega) \leq v_{0}$,

$$
\lambda_{1}(\Omega) \geq \Lambda(\mu(\Omega))
$$


where $\Lambda$ is a non-negative non-increasing function. Let $u(x)$ denote a nonnegative function defined on the vertex set of $\Gamma$ with finite support. We denote

$$
\int_{V} u d \mu=\alpha, \quad \text { and } \quad \int_{V} u^{2} d \mu=\beta .
$$

Then for any $s \geq s_{0}=\alpha / v_{0}$, we have

$$
\int_{E}|\nabla u|^{2} d \sigma \geq(\beta-2 s \alpha) \Lambda\left(s^{-1} \alpha\right)
$$

Now we are ready to state the following lower bounds for $\lambda_{k}$ for large $k$.

Theorem 7.3. In a weighted graph $(\Gamma, \sigma)$, we assume that

$$
\sigma(\partial \Omega) \geq c \mu(\Omega)^{1-1 / n}
$$

for any $\Omega$ satisfying $\mu(\Omega) \leq v_{0}$, where $c>0, v_{0}>0$ and $n>1$. Then the Dirichlet eigenvalue $\lambda_{k}(\Omega)$ satisfies

$$
\lambda_{k}(\Omega) \geq a\left(\frac{k}{\mu(\Omega)}\right)^{2 / n}
$$

for $a=e^{-2 / n}(n+2)^{1-2 / n} c^{2}$, provided

$$
|\Omega| \geq k \geq \frac{(n+2) e}{v_{0}} \mu(\Omega) .
$$

The proof goes the same way as that of Theorem 3.1. The latter used certain properties of the heat kernel and the Laplace operator which all are available in the case of a graph as was shown in Section 5. The constant $a$ is twice as big as one in Theorem 3.1 because we use Proposition 7.1 instead of (3.8).

Combining the above theorem with Theorem 6.3, we obtain the following result.

Corollary 7.4. Assume that the weighted graph $(\Gamma, \sigma)$ satisfies the hypotheses of Theorem 6.3. Then, for any finite set $\Omega \subset V$, the Dirichlet eigenvalue $\lambda_{k}(\Omega)$ satisfies

$$
\lambda_{k}(\Omega) \geq a\left(\frac{k}{\mu(\Omega)}\right)^{2 / n}
$$


provided

$$
|\Omega| \geq k \geq \kappa \frac{\mu(\Omega)}{\omega R_{0}^{n}},
$$

where $\kappa=c_{1}(n) \iota^{n}>0$ and $a=c_{2}(n) \iota^{-2} \nu_{R_{0}+1}^{-2} \omega^{\prime 2} \omega^{2 / n-2}>0$.

Corollary 7.5. For the lattice graph on $\mathbb{Z}^{n}$, for any finite subset $\Omega$ of vertices, we have

$$
\lambda_{k}(\Omega) \geq a\left(\frac{k}{\mu(\Omega)}\right)^{2 / n},
$$

where $k$ is any integer between 1 and $|\Omega|$ and $a=a(n)>0$.

Similarly to Theorem 4.1, we have the following Sobolev inequality.

Theorem 7.6. In a weighted graph $(\Gamma, \sigma)$, suppose that for a finite subset $\Omega$ of vertices with

$$
\mu(\Omega) \leq v_{0},
$$

the following holds

$$
\sigma(\partial \Omega) \geq c \mu(\Omega)^{1-1 / n}
$$

for some $c, v_{0}>0$ and $n>1$. Then, for any function $f$ with finite support, we have

$$
\int_{E}|\nabla f| d \sigma+c \Theta\left(\Omega_{0}\right) \int_{V}|f| d \mu \geq c 2^{-1 / n}\left[\int_{V}|f|^{\frac{n}{n-1}} d \mu\right]^{\frac{n-1}{n}}
$$

where $\Omega_{0}=\{x \in M:|f(x)|>0\}$ and

$$
\Theta(\Omega):= \begin{cases}0, & \text { if } \mu(\Omega) \leq v_{0}, \\ v_{0}^{-1} \mu(\Omega)^{1-1 / n}, & \text { if } \mu(\Omega)>v_{0} .\end{cases}
$$

Furthermore, for any $p \in[1, n)$,

$$
\begin{aligned}
& C\left[\int_{E}|\nabla f|^{p} d \sigma\right]^{1 / p}+c \Theta\left(\Omega_{0}\right)\left[\int_{V}|f|^{p} d \mu\right]^{1 / p} \\
& \geq c 2^{1 / p-1-1 / n}\left[\int_{V}|f|^{\frac{p n}{n-p}} d \mu\right]^{\frac{n-p}{n p}}
\end{aligned}
$$

where $C=C(n, p)>0$. 


\section{References.}

[1] E. Bombieri and E. Giusti, Harnack's inequality for elliptic differential equations on minimal surfaces, Invent. Math. 15 (1972) 24-46.

[2] E. Bombieri, E. de Giorgi, and M. Miranda, Una maggiorazioni a-priori relative alle ipersuperfici minimali non parametriche, Arch. Rat. Mech. Anal. 32 (1969) 255-367.

[3] G. Carron, Inégalités isopérimétriques de Faber-Krahn et conséquences, in Actes de la table ronde de géométrie différentielle en l'honneur de Marcel Berger, Collection SMF Séminaires et Congrès , 1, 1994.

[4] I. Chavel, Eigenvalues in Riemannian geometry, Academic Press, New York, 1984 .

[5] I. Chavel, Large time heat diffusion on Riemannian manifolds, Lecture notes, 1998.

[6] J. Cheeger, A lower bound for the smallest eigenvalue of the Laplacian, in Problems in Analysis: A Symposium in honor of Salomon Bochner, Princeton University Press, Princeton, 1970, 195-199.

[7] J. Cheeger and S.-T. Yau, A lower bound for the heat kernel, Comm. Pure Appl. Math. 34 (1981) 465-480.

[8] S.Y. Cheng and P. Li, Heat kernel estimates and lower bounds of eigenvalues, Comment. Mat. Helv. 56 (1981) 327-338.

[9] F.R.K. Chung and S.-T. Yau, Eigenvalues of graphs and Sobolev inequalities, Combinatorics, Probability and Computing 4 (1995) 11-26.

[10] F.R.K. Chung, Spectral Graph Theory, AMS publications, 1996.

[11] J. Dodziuk, Maximum principle for parabolic inequalities and the heat flow on open manifolds, Indiana Univ. Math. J. 32 (1983) 703-716.

[12] J. Dodziuk, Difference equations, isoperimetric inequalities and transience of certain random walks, Trans. Amer. Math. Soc. 284 (1984) 787-794.

[13] H. Federer, Geometric measure theory, Springer, Berlin, 1969.

[14] H. Federer and W.H. Fleming, Normal and integral currents, Ann. Math. 72 (1960) 458-520.

[15] A. Grigor'yan, Heat kernel upper bounds on a complete non-compact manifold, Revista Mathemática Iberoamericana 10 (1994) 395-452. 
[16] A. Grigor'yan, Estimates of heat kernels on Riemannian manifolds, Spectral Theory and Geometry, Edinburgh, 1998, London Math. Soc. Lecture notes $\underline{273}$, p. $140-225$.

[17] A.K. Gushchin, On the uniform stabilization of solutions of the second mixed problem for a parabolic equation, (in Russian), Matem. Sbornik 119(161) (1982) 451-508. Engl. transl. Math. USSR Sb. 47 (1984) 439-498.

[18] E. Hebey, Sobolev spaces on Riemannian manifolds, Lecture Notes in Math. 1635, Springer, 1996.

[19] D. Hoffman and J. Spruck, Sobolev and isoperimetric inequalities for Riemannian submanifolds, Comm. Pure Appl. Math. 27 (1974) 715-727. See also " $A$ correction to: Sobolev and isoperimetric inequalities for Riemannian submanifolds", Comm. Pure Appl. Math. 28 (1975) 765-766.

[20] P. Li and S.-T. Yau, On the Schrödinger equation and the eigenvalue problem, Comm. Math. Phys. 88 (1983) 309-318.

[21] E. Lieb, The number of bound states of one-body Schrödinger operators and the Weyl problem, Proc. Sym. Pure Math. 36 (1980) 241-252.

[22] V.G. Maz'ya, Classes of domains and embedding theorems for functional spaces, (in Russian), Dokl. Acad. Nauk SSSR 133 (1960) 527-530. Engl. transl. Soviet Math. Dokl. 1 (1961) 882-885.

[23] V.G. Maz'ya, Sobolev spaces (in Russian), Izdat. Leningrad Gos. Univ. Leningrad, 1985, Engl. transl. Springer, 1985.

[24] V.G. Maz'ya, Classes of domains, measures and capacities in the theory of differentiable functions, Encyclopaedia Math. Sci. 26, Springer, Berlin, 1991, 141-211.

[25] J.H. Michael and L.M. Simon, Sobolev and mean-value inequalities on generalized submanifolds of $R^{n}$, Comm. Pure Appl. Math. 26 (1973) 361-379.

[26] J. Nash, Continuity of solutions of parabolic and elliptic equations, Amer. J. Math. 80 (1958) 931-954..

[27] R. Osserman, A survey of minimal surfaces, Dover, New York, 1986.

[28] G. Pólya, On the eigenvalues of vibrating membranes, Proc. London Math. Soc. (3) 11 (1961) 419-433.

[29] L. Saloff-Coste, Isoperimetric inequalities and decay of iterated kernels for almost-transitive Markov chains, Combinatorics, Probability, Computing 4 (1995) 419-442. 
[30] L. Saloff-Coste, Lectures on finite Markov chaines Lecture Notes in Math. 1665, Springer, 1997.

[31] R. Schoen and S.-T. Yau, Lectures on Differential Geometry, Conference Proceedings and Lecture Notes in Geometry and Topology 1, International Press, 1994.

[32] N.Th. Varopoulos, Long range estimates for Markov chains, Bull. Sc. Math., 22ème série 109 (1985) 113-119.

ReCEIVEd OCTOBER 6, 1998.

University of California, San Diego

LA Jolla, CALIFornia 92093-0112

USA

IMPERIAL COLLEGE

LONDON SW7 2BZ

UNITED KINGDOM

AND

HARVARD UNIVERSITY

CAMBRIDGE MA 02138

USA

E-mail addresses: fan@ucsd.edu

a.grigoryan@ic.ac.uk yau@math.harvard.edu 